\title{
Addressing incomplete lineage sorting and paralogy in the inference of uncertain salmonid phylogenetic relationships
}

\author{
Matthew A Campbell ${ }^{\text {Corresp., } 1}$, Thaddaeus J Buser ${ }^{2}$, Michael E Alfaro ${ }^{3}$, J. Andres Lopez ${ }^{1,4}$ \\ 1 University of Alaska Museum, University of Alaska - Fairbanks, Fairbanks, Alaska, United States of America \\ 2 Department of Fisheries and Wildlife, Oregon State University, Corvallis, Oregon, United States of America \\ 3 Department of Ecology and Evolutionary Biology, University of California, Los Angeles, Los Angeles, California, United States of America \\ ${ }^{4}$ School of Fisheries and Ocean Sciences, University of Alaska - Fairbanks, Fairbanks, Alaska, United States of America \\ Corresponding Author: Matthew A Campbell \\ Email address: macampbell2@alaska.edu
}

Recent and continued progress in the scale and sophistication of phylogenetic research has yielded substantial advances in knowledge of the tree of life; however, segments of that tree remain unresolved and continue to produce contradicting or unstable results. These poorly resolved relationships may be the product of methodological shortcomings or of an evolutionary history that did not generate the signal traits needed for its eventual reconstruction. Relationships within the euteleost fish family Salmonidae have proven challenging to resolve in molecular phylogenetics studies in part due to ancestral autopolyploidy contributing to conflicting gene trees. We examine a sequence capture dataset from salmonids and use alternative strategies to accommodate the effects of gene tree conflict based on aspects of salmonid genome history and the multispecies coalescent. We investigate in detail three uncertain relationships: (1) subfamily branching, (2) monophyly of Coregonus, and (3) placement of Parahucho. Coregoninae and Thymallinae are resolved as sister taxa, although conflicting topologies are found across analytical strategies. We find inconsistent and generally low support for the monophyly of Coregonus, including in results of analyses with the most extensive dataset and complex model. The most consistent placement of Parahucho is as sister lineage of Salmo. 
1 Addressing incomplete lineage sorting and paralogy in the inference of uncertain salmonid

2 phylogenetic relationships

3

4 Matthew A. Campbell ${ }^{1, *}$, Thaddaeus J. Buser ${ }^{2}$, Michael E. Alfaro ${ }^{3}$, and J. Andrés López ${ }^{1,4}$ 5

6 Addresses

$7{ }^{1}$ University of Alaska Museum, University of Alaska - Fairbanks, Fairbanks, AK 99775, USA

$8{ }^{2}$ Department of Fisheries and Wildlife, Oregon State University, Corvallis, OR 97331 USA

$9{ }^{3}$ Department of Ecology and Evolutionary Biology, University of California Los Angeles, Los

10 Angeles, CA 90095 USA

$11{ }^{4}$ School of Fisheries and Ocean Sciences, University of Alaska-Fairbanks, Fairbanks, AK

1299775, USA.

13

14

15 *Corresponding Author: DrMacCampbell@gmail.com

16 


\section{ABSTRACT}

18 Recent and continued progress in the scale and sophistication of phylogenetic research has 19 yielded substantial advances in knowledge of the tree of life; however, segments of that tree

20 remain unresolved and continue to produce contradicting or unstable results. These poorly

21 resolved relationships may be the product of methodological shortcomings or of an evolutionary

22 history that did not generate the signal traits needed for its eventual reconstruction. Relationships

23 within the euteleost fish family Salmonidae have proven challenging to resolve in molecular

24 phylogenetics studies in part due to ancestral autopolyploidy contributing to conflicting gene

25 trees. We examine a sequence capture dataset from salmonids and use alternative strategies to

26 accommodate the effects of gene tree conflict based on aspects of salmonid genome history and

27 the multispecies coalescent. We investigate in detail three uncertain relationships: (1) subfamily

28 branching, (2) monophyly of Coregonus, and (3) placement of Parahucho. Coregoninae and

29 Thymallinae are resolved as sister taxa, although conflicting topologies are found across

30 analytical strategies. We find inconsistent and generally low support for the monophyly of

31 Coregonus, including in results of analyses with the most extensive dataset and complex model.

32 The most consistent placement of Parahucho is as sister lineage of Salmo.

\section{KEYWORDS}

35 Coregonus - Gene Tree and Species Tree Conflict - Incomplete Lineage Sorting - Parahucho 36 Salmonidae - Segmental Polyploidy 


\section{INTRODUCTION}

38 Within ray-finned fishes (Actinopterygii), there are numerous relationships that are challenging to resolve. Historically, anatomical characteristics have provided great insight into the evolutionary relationships of some groups, but failed to confidently resolve others (e.g., Betancur-R et al., 2017). In the past few decades, DNA sequence data has emerged as a method to gather numerous characters for phylogenetic inference. Sequence data generation in recent years has undergone dramatic and rapid evolution with respect to the cost and scale of DNA sequence determination and has fostered the production of genomic-scale datasets for phylogenetic estimation. The theory and implementations of methods for analyzing these data have increased in complexity and performance broadly in parallel with increased data generation (Muir et al., 2016). The sheer quantity of data contained within genome-scale datasets has contributed to expectations that molecular phylogenetics may provide conclusive results regarding previously elusive evolutionary relationships. While additional anatomical investigation and the application of molecular phylogenetics has aided the resolution of some groups, such as the Scorpaenoidea (e.g., Smith et al., 2018), counterpoints certainly remain. In particular, there are parts of the ray-finned fish tree of life that remain poorly resolved even with the application of high-throughput sequencing data sets of substantial numbers of loci. These persistently challenging-to-resolve relationships include those among early-branching euteleost lineages (e.g., Campbell et al., 2017) and the intra-clade relationships of Pelagiaria (e.g., Campbell et al., 2018; Friedman et al., 2019; Miya et al., 2013).

While conclusive resolution is desirable, evolutionary relationships can be expected to remain unresolved with molecular data under certain scenarios. For example, when the characteristics of the evolutionary process being investigated generated signals with ambiguous origins, prevented the formation and preservation of a phylogenetic signal, or when the methodological framework used to investigate that process is poorly suited for the data type. Discordance between gene trees or gene tree - species tree (GT-ST) conflict (Figure 1) represents a special challenge in the inference of recalcitrant relationships because it may be the combined product of conflicting histories and functionally undetectable phylogenetic signal with difficult to disentangle contributions from each factor. 
68 One predicted cause of GT-ST conflict is incomplete lineage sorting (ILS), which may be

69 defined as the failure for two or more allelic lineages to coalesce within a population (Degnan

70

71

72 and Rosenberg, 2009). Incompletely sorted diversity can lead to conflicting gene tree topologies if the time between speciation events is small and/or the population sizes are large (Figure 2). The continued investigation of natural systems has emphasized the pervasiveness of hybridization (Mallet, 2005), and divergence times before or after hybridization may result in ILS and gene tree incongruence signatures identical to patterns exhibited by hybridization alone (Yu et al., 2011). In tree-based evolutionary analyses, hybridization events are very easily overlooked, and GT-ST conflict will be considered a consequence of ILS. Alternatively, in phylogenetic network analysis, such reticulations are largely taken as hybridization (Yu et al., 2011). Another potential source of GT-ST conflict is gene or genome duplication events. Genes that are duplicated, particularly those arising from whole-genome duplication (WGD) are frequently lost (e.g. Berthelot et al., 2014; Scannell et al., 2007). However duplication of a gene followed by loss of alternative copies in different lineages may lead to GT-ST conflict (Figure 3). While massive gene loss is typical following WGD as polyploid organisms revert to a normal ploidy level (Gerstein and Otto, 2009), many genes in the genomes of lineages with ancestral genome duplications exist in a continuum from polyploidy to normal ploidy, a condition known as segmental polyploidy. In lineages where genomes exhibit segmental polyploidy, ILS can be expected to be more pronounced in the tetrasomic regions of genome compared to the disomic regions as their respective population sizes differ (Figure 2B \& 2D).

Whole-genome duplication events are known from across many eukaryotic lineages (e.g. Campbell et al., 2016) and some lineages have experienced successive rounds of WGD producing nested levels of ancestral polyploidy in the genomes of descendant lineages (Meyers et al., 2006; Wendel, 2015). Genomes of extant fishes are partly shaped by two rounds of WGD (commonly noted as 1R and 2R; Supplemental Figure S1) which are inferred to have taken place 450-600 million years ago (mya) (Dehal and Boore, 2005). A third well-supported WGD (3R) is inferred in the common ancestor of teleosts at around 350 mya (Meyer and Van de Peer, 2005). Additional WGD events are scattered through lineages in the fish tree of life (e.g. Leggatt and Iwama, 2003). A WGD occurred in the common ancestor of the Salmoniformes some time after the lineage diverged from the Esociformes. This WGD event occurred $~ 88$ mya ago and it is 
99 known as the Ss4R (salmonid-specific fourth round) (Berthelot et al., 2014; Macqueen and

100 Johnston, 2014).

101

102 Salmonids are a group of 228 fish species divided among three subfamilies: Coregoninae

103 (whitefishes, ciscos, and innocu: 86 species), Thymallinae (graylings: 18 species), and

104 Salmoninae (salmons, trouts, charrs, taimens: 124 species) (Fricke et al., 2019; Nelson, 2006).

105 Despite the commercial and cultural importance of many salmonids, and intensive research on

106 many of the commercially exploited species, questions about their evolutionary relationships

107 remain unanswered. While monophyly of each of the three subfamilies is generally accepted,

108 relationships between these subfamilies remain contentious. A sister-group relationship between

109 Salmoninae and Thymallinae $(\mathrm{S}+\mathrm{T})$ has been supported by morphological (Sanford, 1990;

110 Wilson and Williams, 2010) and molecular evidence (Betancur-R et al., 2013; Shedko et al.,

111 2012; Yasuike et al., 2010). In contrast, several molecular phylogenetic studies have offered

112 support for a clade comprising Coregoninae and Salmoninae (C + S) (Alexandrou et al., 2013;

113 Crête-Lafrenière et al., 2012; Near et al., 2012). Finally, Coregoninae sister to Thymallinae (C +

$114 \mathrm{~T}$ ) has been indicated by several analyses (Burridge et al., 2012; Campbell et al., 2013; Horreo,

115 2017; Li et al., 2010; Macqueen and Johnston, 2014; Robertson et al., 2017). Thus, each of the

116 three possible arrangements of the subfamilies has found some support in the literature. Another

117 point of contention is the relationship between the coregonine genera Coregonus and Stenodus.

118 Previous studies have variously placed Stenodus as sister to (Bodaly et al., 1991; Horreo, 2017;

119 Sajdak and Phillips, 1997; Vuorinen et al., 1998) or nested within Coregonus (Bernatchez et al.,

120 1991a; Crête-Lafrenière et al., 2012). A final point of debate is the placement of Parahucho

121 within Salmoninae. Previous studies have variously placed Parahucho in a polytomy with

122 Oncorhynchus + Salmo + Salvelinus (Shedko et al., 2012) or as sister to: the genus Salmo

123 (Crespi and Fulton, 2004; Lecaudey et al., 2018; Matveev et al., 2007; Oakley and Phillips,

124 1999); the genus Salvelinus (Campbell et al., 2013; Crête-Lafrenière et al., 2012; Horreo, 2017);

125 a clade composed of Oncorhynchus + Salvelinus (Crête-Lafrenière et al., 2012); or a clade

126 composed of Salmo + Salvelinus + Oncorhynchus (Alexandrou et al., 2013).

127

128 While phylogenetic uncertainty is not unique to salmonids, the numerous hypotheses of salmonid

129 intrarelationships proposed in recent literature suggest serious challenges with molecular 
130 phylogenetic inference of the group and WGD may be a contributing factor (Allendorf and

131 Thorgaard, 1984; Berthelot et al., 2014; Lien et al., 2016; Macqueen and Johnston, 2014; Ohno,

132 1970). Well-characterized genomes of salmonids reveal that $10-15 \%$ of their genomes are

133 tetrasomically inherited, with high levels of overall genomic similarity throughout as a result of

134 the Ss4R (e.g., Lien et al., 2016; Pearse et al., 2019). Within rainbow trout (Oncorhynchus

135 mykiss), for example, these duplicated regions contain 2,278 tetrasomically inherited pairs of

136 genes (Campbell et al., 2019; Pearse et al., 2019), meaning that $10.62 \%$ of the 42,884 annotated

137 protein-coding genes in rainbow trout are tetrasomically inherited. Comparative genome

138 mapping shows broad conservation of partial tetrasomy across Salmoninae in eight chromosome

139 arms, the "Magic Eight" with homologous regions in Salmo, Salvelinus, and Oncorhynchus

140 species indicated to be tetrasomic (Brieuc et al., 2014; Campbell et al., 2019; Kodama et al.,

141 2014; Lien et al., 2011; Sutherland et al., 2016). Further analyses of recently produced genome

142 assemblies and linkage mapping has continued to support the idea of the same genomic regions

143 maintaining tetrasomy across salmonids (Blumstein et al., 2019). Genomic comparisons support

144 that there are at least seven tetrasomically pairing chromosomes in salmonids, with possible

145 intermediate pairs falling between tetrasomy and disomy present, and one tetrasomically pairing

146 set identified only by linkage mapping. Tetrasomic recombination results in larger population

147 sizes and duplicated loci for some genes and genome regions and may contribute to GT-ST

148 conflict via ILS (Figure 2B \& 2D). While the path to rediploidization in salmonids has conserved

149 patterns across species where rediploidization occurred prior to speciation, continued

150 rediploidization within lineages after speciation has resulted in unique signatures - lineage-

151 specific rediploidization or lineage-specific ohnologue resolution (LORe). These signatures are

152 elevated in tetrasomically pairing regions of the salmonid genome (Robertson et al. 2017). Due

153 to LORe, loci from tetrasomically pairing genomic regions of salmonid genomes are likely to

154 have problematic orthology assignment. With a complex genome structure riddled with

155 duplications and high-similarity regions that are undergoing independent rediploidization, the

156 potential for the accidental inclusion of paralogous loci in phylogenetic analyses of this group is

157 concerning (i.e. Figure 3). Together both tetrasomy and lineage-specific rediploidization

158 processes may be responsible for widespread GT-ST conflict in salmonids. 
160 In this paper we analyze a DNA sequence dataset from 500 Ultra Conserved Element (UCE)

161 loci. These UCE loci were designed to be exchangeable across data sets and to be single copy

162 across actinopterygiian fishes (Faircloth et al. 2013). One particular property of UCE loci that is

163 notable is that variation increases away from the core conserved region, providing variable sites

164 across time scales and higher phylogenetic informativeness compared to protein-coding gene loci

165 (e.g., Gilbert et al., 2015). The generous starting pool of 500 loci allows us to filter for paralogs

166 or duplicates originating from the Ss4R while retaining relatively informative loci for

167 phylogenetic inference. With UCE loci from representative salmonids, we test the following

168 phylogenetic hypotheses while considering particularities of salmonid genome evolution: (1), the

169 relationships of salmonid subfamilies, (2), the monophyly of the coregonine genus Coregonus,

170 and (3), the placement of the enigmatic salmonine genus Parahucho. To identify clear orthologs

171 for analysis within salmonids from markers designed from diploid species, we filter loci based

172 on assembled duplicates and on genomic location in known tetrasomic regions of salmonid

173 genomes. We then test the effects of missing data and tetrasomic inheritance in phylogenetic

174 analyses by generating alternative subsamples of the full dataset and compared the resulting

175 inferred relationships with and without the multispecies coalescent.

\section{METHODS}

\section{Sequence data acquisition and processing}

179 We obtained sequence data from representatives of all major salmonid genera $(n=15)$, as well as

180 from representatives of the two extant esociform families Esocidae and Umbridae, which are the 181 closest diploid relatives of salmonids (Ishiguro et al., 2003; López et al., 2004). We generated

182 genetic sequence data for phylogenetic analysis by sequence capture of 500 UCE loci following

183 the protocol outlined for the 500 UCE actinopterygiian probe set (Faircloth et al., 2013) modified

184 according to experimental conditions described in Campbell et al. (2017). We sequenced these

185 loci for 11 taxa and incorporated data for six additional taxa from previous studies described in

186 Supplemental Table S1 (Campbell et al., 2017; Faircloth et al., 2013).

188 Demultiplexed reads were trimmed of low-quality bases and filtered for minimum length with

189 Trimmomatic version 0.32 using the following command line specifications and adapter fasta

190 file available for download with the program: ILLUMINACLIP: TruSeq3-PE- 
191 splitAdapter.fa:2:30:10 LEADING:3 TRAILING:3 SLIDINGWINDOW:4:15 MINLEN:36

192 (Bolger et al., 2014). Sequences from a subset of samples were assembled with Velvet version

193 1.2.10 (Zerbino and Birney, 2008) to establish a range of suitable k-mer lengths for sequence

194 assembly. We initially attempted optimal assemblies using various criteria with VelvetOptimiser

195 version 2.2.5 (Gladman and Seemann, 2012). However, because some regions of the salmonid

196 genome are tetrasomic, and thus do not meet the ploidy assumptions made in VelvetOptimiser,

197 we established a range of k-mer lengths, 57 to 83 sites representing between 1/2 and 2/3 of total

198 read length, and assembled salmonid sequences from all odd k-mer values within that range. We

199 evaluated each of the resulting assemblies for the presence of UCEs using the PHYLUCE

200 pipeline (Faircloth, 2015) and retained only the assembly that contained the maximum number of

201 UCE loci, following the approach described in Campbell et al. (2017).

202

203 We identified homologous UCE loci and prepared them for alignment using the PHYLUCE

204 pipeline. The PHYLUCE package screens out reciprocally duplicate enriched loci (i.e., potential

205 paralogs) and performs a multiple sequence alignment of each of the remaining loci using

206 MAFFT version 7.130b (Katoh et al., 2002; Katoh and Standley, 2013; Katoh and Toh, 2008).

207 We screened tetrasomic loci in our dataset by searching previously published assemblies of

208 Atlantic salmon (Salmo salar) (Lien et al., 2016) and rainbow trout (Pearse et al., 2019) for the

209 UCEs sequenced in our study. Within Atlantic salmon, nine pairs of chromosome arms were

210 indicated by Lien et al. (2016) that may be tetrasomic, seven of which were identified LORe

211 regions by Robertson et al. (2017). From rainbow trout, seven chromosome pairs that were

212 clearly tetrasomic in Pearse et al. (2019) were screened constituting homeologs to the seven

213 LORe pairs screened in Atlantic salmon (Blumstein et al., 2019). BLASTN (Altschul et al.,

214 1990) alignments of assembled UCEs that presented two alignments of $\geq 95 \%$ overlap and $\geq 97 \%$

215 similarity in the tetrasomic regions of either Atlantic salmon or rainbow trout were considered

216 tetrasomic. UCEs that were not assembled and found to be single copy for all targeted species

217 were also removed to create a "100\% coverage alignment."

218

219 To understand the effects of relaxing strict filtering of loci on phylogenetic inference, we

220 generated four additional datasets: 1) a "95\% coverage alignment" allowing one missing taxon

221 per locus, 2) a "93\% coverage alignment" allowing up to two missing taxa per locus, 3) a "75\% 
222 coverage alignment" allowing up to five missing taxa per locus, 4) a "tetrasomic loci alignment"

223 including only tetrasomically inherited loci permitting 5 missing taxa per locus.

224

225

\section{Phylogenetic inference with the multispecies coalescent}

226

227

228

229

230

231

232

233

234

235

236

237

238

239

240

241

242

243

244

245

246

247

248

249

250

251

252

We conducted joint GT-ST estimation of each of the five datasets with the StarBEAST2 Bayesian framework (Ogilvie et al., 2017) as implemented in BEAST version 2.4.8 (Bouckaert et al., 2014). For analysis in StarBEAST2, our data sets were partitioned by each UCE locus and the Akaike Information Criterion (AIC) was applied to select the best-fitting model of nucleotide substitution for each locus in each of our datasets using ModelGenerator version 0.85 (Keane et al., 2006). We ran several iterations of BEAST with varying length and sampling frequency and evaluated convergence of the runs and effective sample sizes (ESS) with Tracer version 1.6 (Rambaut et al., 2018) to determine proper sampling frequency and MCMC chain run length. We required that ESS values be 200 or greater to assure an adequate sample size for our posterior parameter estimations. Analysis of our initial BEAST runs showed that the estimates of parameter values failed to converge for datasets with more than 13 UCEs even after 300 million to 2 billion MCMC generations and showed evidence of over-parameterization, such as a lack of convergence in each chain and low consistency in $\mathrm{LnL} /$ prior values. To account for this lack of convergence in the larger datasets and test the effects of using a simpler nucleotide substitution model, we conducted an additional set of phylogenetic inferences in BEAST of all of our datasets and partition schemes thereof but specified the $\mathrm{HKY}+\Gamma$ nucleotide substitution model with four categories of gamma distributed rate variation and empirical base frequencies for each partition. For each of our analyses, we combined the independent chains and applied a burnin with LogCombiner version 2.1.3, and generated a maximum clade credibility tree using TreeAnnotator version 2.1.2, specifying a posterior probability (PP) limit of 0.50 and median node heights.

In addition to the joint GT-ST estimation conducted in BEAST, we also estimated the ML tree of each UCE locus and used summary coalescence analyses to estimate a species tree from the resulting set of gene trees a posteriori. We conducted this analysis in addition to the joint GT and ST analysis conducted in StarBEAST2 because this approach can show high accuracy and is computationally more tractable than a full probabilistic approach (Mallo and Posada, 2016). For

Peer) reviewing PDF | (2019:11:42789:1:1:NEW 16 May 2020) 
253 each of our datasets, we estimated the ML tree of each UCE locus using RAxML version 8.0.19,

254 specifying the GTR $+\Gamma$ model of sequence evolution. We passed these trees to ASTRAL version

255 5.5.9 and MP-EST version 2.2.0, which each estimated a species tree from the collection of UCE 256 loci.

257

258 Concatenated analyses

259 A concatenated maximum likelihood (ML) analysis was conducted with each data set (100\%,

$26095 \%, 93 \%, 75 \%$ coverage alignments and the tetrasomic loci alignment). We conducted both

261 partitioned (i.e., by gene, or objectively defined) and un-partitioned analyses under the

$262 \mathrm{GTR}+\Gamma$ model of sequence evolution using RAxML version 8.2.10 (Stamatakis, 2014) with

263 rapid bootstrap stopping. We used the program PartitionFinder version 1.1.1 (Lanfear et al.,

264 2012) to generate an objective optimal partitioning strategy with partitions of individual UCE

265 loci specified with the following options: the GTR $+\Gamma$ model, greedy algorithm (Lanfear et al.,

266 2014) and likelihoods estimated by RAxML (--raxml option). If the objective partition strategy

267 differed from either unpartitioned or partitioned strategies, a third analyses of that data set was

268 undertaken with RAxML.

269

270 Salmonid subfamily relationships

271 The composition and monophyly of salmonid subfamilies is well-established (e.g., Norden,

272 1961; Sanford, 1990). Nonetheless, relationships between the three salmonid subfamilies remain

273 contentious although there are only three possible arrangements of these taxa in a bifurcating tree

274 if the subfamilies truly are monophyletic. This tractable number of potential combinations allows

275 us to consider each possible arrangement of the taxa explicitly and test for the most preferred

276 arrangement given our data. To avoid test-specific biases, we evaluated the support for each

277 possible relationship of the subfamilies using four different approaches: (1) triplet analysis of

278 independently estimated gene trees, (2), Bayes factors computed from the posterior trees of

279 StarBEAST2 analyses, (3), a test under the multi-species coalescent with the program MP-EST,

280 and, (4), approximately unbiased (AU) tests of concatenated trees.

281

282 1. Triplet analysis 
283 A triplet analysis is based around a three-taxon rooted tree. In this manuscript, the three salmonid

284 subfamilies form the basis of the test. Expectations with triplet analyses are that given the three

285 possible relationships present with a rooted three-taxon case, the correct relationship should

286 predominate over roughly equal portions of the alternative hypotheses (Campbell et al., 2014;

287 Cranston, 2010; Pamilo and Nei, 1988). We performed triplet analysis using only the most

288 stringent (i.e., no tetrasomic loci, no missing taxa) dataset, the $100 \%$ coverage alignment. For

289 each UCE locus, a salmonine, coregonine, and thymalline were chosen at random with Esox

290 lucius as a fixed outgroup. We inferred a ML for each set of taxa with RAxML and the tree

291 stored. We repeated the process of random draws of three taxon sets 1,000 times for each locus

292 and calculated the final frequency of relationships among the three salmonid subfamilies.

293

294 2. Bayes factors

295 We compared the strength of evidence for different salmonid subfamily relationships using

296 Bayes factors calculated from the StarBEAST2 post-burnin trees (Jeffreys, 1935; Kass and

297 Raftery, 1995). We assumed a uniform prior for these calculations, that is, each of the three

298 subfamily relationships were equally likely. A Bayes factor is then the ratio of the posterior

299 probabilities between two alternatives, and here, we present ratios of posterior probabilities of

$300 \mathrm{C}+\mathrm{T} / \mathrm{C}+\mathrm{S}, \mathrm{C}+\mathrm{T} / \mathrm{S}+\mathrm{T}$ and $\mathrm{C}+\mathrm{S} / \mathrm{S}+\mathrm{T}$.

301

302 3. Tests under multispecies coalescent

303 We used a likelihood ratio test, incorporating the multispecies coalescent with MP-EST version

304 2.2.0 (Liu et al., 2010) and the R package phybase version 2.0 (Liu and Yu, 2010) available at

305 https://github.com/lliu1871/phybase. We supplied unconstrained gene trees from our summary

306 coalescence analyses to MP-EST and constrained the species tree topologies but allowed the

307 branch lengths to be optimized to best fit the data presented by the gene trees. We modified the

308 test2.sptree function of phybase (provided in Data Supplement,

309 https://doi.org/10.25338/B8DC81) to enable all three subfamily arrangements to be compared as

310 described in Liu et al. (2019). Likelihood ratio test statistics $(t)$ were calculated between pairs of

311 alternative trees and we generated a distribution of the test statistic by bootstrap sampling gene

312 trees one hundred times to estimate the null distribution for $t$ and provide a measure of statistical

313 significance $(p-$ value). 


\section{4. Approximately unbiased tests}

316 We conducted topological tests of the three possible relationships of salmonid subfamilies in a

317 concatenated framework by constraining subfamily relationships for different alignments and

318 partitioning schemes and passing per site likelihoods from RAxML version 8.2.11 to the

319 program CONSEL version 0.20. We used CONSEL to compute the AU test from these per site 320 likelihoods (Shimodaira and Hasegawa, 2001).

\section{RESULTS}

\section{Sequence alignment characteristics}

324 Of the 500 UCE loci that we targeted, the number of total captured loci and the number of 325 putatively single copy loci sequenced from each taxon varied (Supplemental Table S1). For 326 diploid outgroups, Esox and Umbra, 415 and 409 of the captured UCEs, respectively, were 327 determined to be single-copy. Within salmonids, 166 to 275 of the captured loci were indicated 328 to be single copy. Overall, we retained only eight single-copy UCE loci across all sampled taxa.

329 Two of these loci were identified as coming from tetrasomically-inherited regions of the 330 salmonid genome, and so were removed. Thus only six out of 500 candidate UCE loci passed the 331 most stringent screening process and were present in all targeted taxa for a total of 2,331 aligned

332 nucleotide sites (Table 1). The datasets that we generated when allowing for missing data yielded 333 more retained UCE loci, ranging between 13 and 105, with aligned lengths between 5,297 and 33436,835 aligned nucleotide sites (Table 1). We identified 33 tetrasomically inherited loci for 335 analysis in the "Tetrasomic loci alignment" (see Table 1) and this dataset contains a total of 336 12,758 aligned nucleotide sites. Across all datasets, the proportion of gaps and other 337 ambiguously alignment regions ranged from $15.63 \%$ to $33.83 \%$.

\section{Model selection and MCMC convergence}

340 For each locus in the " $100 \%$ coverage" alignment (see Table 1), we initially specified the model

341 of nucleotide substitution that received the lowest AIC score from ModelGenerator

342 (Supplemental Table S2). We obtained sufficient ESS and convergence of the parameter

343 estimates with three independent MCMC chains of 400 million generations, sampled every

34440,000 generations, with a 10\% burn-in. For the "95\% coverage" dataset, we specified 
345 nucleotide substitution models for each locus which received the lowest AIC score in our

346 analysis using ModelGenerator. We obtained sufficient ESS and convergence with two chains of

34750 million generations, sampled every 5,000 generations, with a 10\% burn-in. For all remaining

348 datasets, analyses with models of nucleotide substitution specified by ModelGenerator failed to

349 generate sufficient ESS and convergence of parameter estimates in our MCMC chains. In

350 response, we broadly reduced parameterization by simplifying nucleotide substitution models.

351

352 Analyses of the "93\% coverage" alignment, the "75\% coverage", and "tetrasomic loci"

353 alignments generated sufficient ESS and convergence of the parameter estimates with two

354 independent MCMC chains of 200 million generations sampled every 20,000 generations, three

355 chains of 800 million generations sampled every 80,000 generations, and four chains of 200

356 million generations sampled every 20,000 generations, respectively, and in each case we applied

357 a $10 \%$ burn-in.

358

359

\section{Phylogenetic inference}

360 The results of joint GT-ST analyses are shown in Figures 4 and 5 and summarized in Table 2.

361 The clade Coregoninae + Thymallinae $(\mathrm{C}+\mathrm{T})$ is found in our 100\% coverage alignment with

362 ModelGenerator-specified nucleotide substitution models with strong support (0.98 PP) and in

363 all analyses with reduced coverage datasets with very weak support ( $0.34-0.52$ PP; see Table

364 2). Analysis of the tetrasomic loci alignment strongly supports Coregoninae + Salmoninae $(\mathrm{C}+$

365 S, 0.99 PP). The monophyly of Coregonus is not supported in the results of the $100 \%$ coverage

366 species-tree, but is weakly supported $(0.43-0.45 \mathrm{PP})$ in two of three of our reduced coverage

367 analyses and strongly supported in our tetrasomic loci species-tree (1.00 PP). Salmo is inferred as

368 the sister group of Parahucho in the $100 \%$ coverage species-tree with strong support (0.99 PP)

369 and in all the reduced coverage analyses $(0.98-0.99 \mathrm{PP})$. In contrast, the species tree inferred

370 from the tetrasomic loci alignment places Oncorhynchus and Parahucho as sister lineages (0.38

371 PP).

372

373 The results of the summary coalescent analyses conducted in ASTRAL support a sister group

374 relationship between Salmoninae and Thymallinae (4/5 analyses), but with low support (PP

375 ranges from 0.38 to 0.57 ), and, from tetrasomic loci, Coregoninae + Salmoninae (0.91 PP, see 
376 Table 2). Monophyly of Coregonus is supported in the results of $3 / 5$ of the analyses, though with

377 only weak support (PP ranges from 0.68 to 0.74). Within Salmoninae, Parahucho is variously

378 supported as sister to several different taxa with weak support $(\mathrm{BPP}<0.70)$, and to a

379 Oncorhynchus + Salvelinus clade with moderate support from tetrasomic loci (0.85 BPP, see

380 Table 2). The results of the summary coalescent analyses conducted in MP-EST are inconsistent

381 and reported in Table 2.

382

383 Concatenated analyses

384 Concatenated ML analyses supported either Coregoninae + Salmoninae or Salmoninae +

385 Thymallinae relationships depending on the number of loci and partitioning strategy

386 (Supplemental Table S3, trees provided in Data Supplement). Partitioning strategy of alignments

387 only altered inferred subfamily relationships in one case (95\% coverage alignment), but caused

388 substantial changes in bootstrap support (BS) values. Monophyly of Coregonus was only

389 supported in three of thirteen analyses (BS range 70-100\%), and the sister of Parahucho varied

390 from Salmo (5/13 analyses, BS range 24-100\%), to Oncorhynchus (5/13 analyses, BS range 50-

$39199 \%)$ to Salvelinus (3/13 analyses, BS range 54-67\%).

392

393 Salmonid subfamily relationships

394 A majority (50.79\%) of trees generated in the triplet analyses support a Coregoninae +

395 Thymallinae clade. The Salmoninae + Coregoninae (31.31\%) and Salmoninae + Thymallinae

$396(17.90 \%)$ clades are found in smaller but substantial proportions of the remaining trees. Bayes

397 factors from the $100 \%$ coverage alignment indicate that a sister group relationship of

398 Coregoninae and Thymallinae should be strongly preferred. Bayes factors for all other datasets

399 when comparing $\mathrm{C}+\mathrm{T}$ to alternatives do not provide strong support for $\mathrm{C}+\mathrm{T}$ (Table 3). MP-

400 EST analysis indicated that a sister group relationship for $\mathrm{C}+\mathrm{S}$ was significantly supported via

401 likelihood ratio tests for the tetratomic loci; no other data sets could distinguish among

402 alternative topologies. The concatenated AU test results were not significant with all 100\% and

$40395 \%$ coverage alignment tests, indicating that the alternative $\mathrm{C}+\mathrm{T}$ hypothesis could not be

404 rejected in analyses of the reduced data sets (Supplemental Table S3).

405

406

\section{DISCUSSION}




\section{Salmonid subfamily relationships}

408 A sister group relationship of Coregoninae and Thymallinae $(\mathrm{C}+\mathrm{T})$ is broadly preferred to 409 alternative topologies in our analyses (e.g., Figure 5). This relationship is recovered in the joint

410 GT-ST estimations of all data sets that exclude tetrasomically-inherited loci $(\mathrm{PP}=0.34-0.98)$

411 and is also the preferred hypothesis indicated by Bayes factors and the triplet analysis. While no

412 concatenated results support $\mathrm{C}+\mathrm{T}$, this result is obtained with as few as six UCE loci in joint

413 GT-ST estimation with declining support as the number of loci increased. A Coregoninae +

414 Thymallinae clade has been identified in previous molecular studies that either implicitly or 415 explicitly account for potential polyploidy in their data. The $\mathrm{C}+\mathrm{T}$ clade was recovered by 416 analyses of mitochondrial genomes, which are not affected by issues of paralogy and can be 417 expected to be less prone to ILS due to their haploid mode of transmission (Campbell et al., 418 2013; Horreo, 2017). Likewise, constructing phylogenies with both copies of Ss4R duplicated 419 genes, an explicit approach, leads to support of a sister Coregoninae + Thymallinae (Macqueen 420 and Johnston, 2014; Robertson et al., 2017).

421

422 Our results do not support the conclusions of previous studies that examined morphological 423 characters of the salmonids and unanimously placed Thymallinae as the sister group to 424 Salmoninae (Kendall and Behnke, 1984; Sanford, 1990; Stearley and Smith, 1993; Wilson and $425 \mathrm{Li}, 1999)$. Furthermore, our results combined with mitogenomic and other studies explicitly 426 addressing polyploidy strongly support $\mathrm{C}+\mathrm{T}$ over the two alternative hypotheses apparent in 427 molecular phylogenetic studies of salmonids (e.g., Alexandrou et al., 2013; Crête-Lafrenière et 428 al., 2012; Shedko et al., 2012). In light of growing molecular evidence conflicting with the 429 morphology-based hypothesis, a critical reassessment of the morphological evidence that 430 supports that relationship is needed but is beyond the scope of the present study.

431

432 Though $\mathrm{C}+\mathrm{T}$ is the preferred grouping in our most stringent analyses, not all of the inference 433 strategies implemented here supported this relationship. Analysis of the concatenated dataset and 434 GT-ST estimation using ASTRAL produced inconsistent results and generally did not support 435 the $\mathrm{C}+\mathrm{T}$ clade. The inconsistent results between summary coalescence approach and joint 436 estimation of the species tree and gene trees may indicate loss of a weak phylogenetic signal in 437 the summarization step. Alternatively, the phylogenetic signal may have been blurred as a result 
438 of treating all UCE loci as a single partition evolving under a common substitution mode. The 439 triplet analysis did not meet our prediction that, given three possible topologies, the true 440 relationship should predominate and the alternative, incorrect arrangements should receive 441 approximately equal portions of support. The triplet analyses yielded the putatively incorrect 442 topologies at unequal frequencies $(31.30 \%$ and $17.90 \%)$. This could be a result of the relatively 443 small size of our " $100 \%$ coverage" alignment or be a product of resampling selecting certain 444 individuals frequently due to few representatives in some subfamilies (two thymallinine species 445 are in the dataset).

\section{Monophyly of Coregonus}

448 Our results inconsistently support the monophyly of Coregonus (e.g., Figures 4 \& 5). Though the 449 joint GT-ST analysis of tetrasomic loci and the ML analysis of the concatenated $100 \%$ coverage data set shows strong support for a monophyletic Coregonus, all other analyses that recovered the clade did so with weak support ( $\mathrm{pp}<0.75, \mathrm{BS}=71 \%$, see Table 2 ). Even within a given analytical framework, support for monophyly of this genus varied. For example, MP-EST analyses showed a monophyletic Coregonus for the two most stringent datasets, but not in the analysis of any others. The uncertainty in this relationship within our data set may be derived from the relatively young age (i.e., 10 mya, Horreo 2017) of the clade that includes Stenodus and Coregonus resulting in low variation in the conserved UCE loci that were indentified to be single copy across a broad range of salmonid species. Additionally, during the analytical study design of this manuscript, a chromosomal-level assembly of a coregonine was unavailable leading to uncertainty in the tetrasomic status of loci in this subfamily based of off salmonine genome assemblies.

Previous studies examining the monophyly of Coregonus with respect to Stenodus have yielded conflicting results (e.g., Bernatchez et al., 1991a; Bodaly et al., 1991; Crête-Lafrenière et al., 2012; Horreo, 2017; Sajdak and Phillips, 1997; Vuorinen et al., 1998). Most recently, analysis of mitogenomic data has shown Coregonus to be monophyletic, with Stenodus as its sister taxon (Horreo 2017). However, the phylogenetic placement of the enigmatic Coregonus huntsmani, a divergent member of the genus, remains untested in any mitogenomic or phylogenomic study. 
469 Crête-Lafrenière et al. (2012) found C. huntsmani to be the sister lineage to Stenodus and all

470 other Coregonus species and earlier isozyme and mitochondrial restriction data show $C$.

471 huntsmani to be distinct from the other main Coregonus subgenera (Coregonus and Leucichthys)

472 but did not include Stenodus in the sampling (Bernatchez et al., 1991b). We examined publicly

473 available COI data from coregonines including two haplotypes from C. huntsmani and found that

474 species to be sister group to a monophyletic Coregonus with low support $(\mathrm{BS}=58 \%$, alignment

475 tree and methods are supplied in the Data Supplement). At present molecular data from $C$.

476 huntsmani are very limited, however including this taxon in future datasets will be critical for

477 conclusively testing the monophyly of Coregonus and its relationship with Stenodus. Further

478 investigations of phylogenetic relationships of Coregonus and Stenodus should also consider the

479 role and prevalence of hybridization within Coregonus and between Stenodus and Coregonus,

480 (e.g., McPhail, 2007) as the signatures of hybridization and ILS are difficult to disentangle and

481 our analyses assumed such signatures derived from ILS (Yu et al., 2011).

482

483 Placement of Parahucho

484 The Sakhalin taimen Parahucho perryi is a rare species and the sole member of its genus (Rand,

485 2006; Vladykov and Gruchy, 1972). This critically endangered fish occupies a limited

486 geographic range and has a narrow range of suitable habitats in Northern Japan and the Russian

487 Far East (Fukushima et al., 2011; Kimura, 1966; Rand, 2006). The placement of Parahucho as

488 sister to Salmo is strongly supported by all joint GT-ST analyses of non-tetrasomic loci (4 of 4,

489 0.98-0.99 PP) and by concatenated analysis of our $100 \%$ and $95 \%$ coverage alignment $(\mathrm{BS}=66-$

$490100 \%$ ). The original description of Sakhalin taimen placed it as a congener of Atlantic salmon

491 (Salmo salar), as Salmo perryi (Brevoort, 1856). Subsequently, the similarities of Sakhalin

492 taimen to the European huchen Hucho hucho were noted, and these two species were placed in

493 the same genus, Hucho (Günther, 1868; Jordan and Snyder, 1902). Hucho and Parahucho are

494 both large bodied piscivores, but differ in that the latter has 10 less vertebrae, basibranchial teeth,

495 a median set of teeth on the supralingual, and 70-100 less scales in the lateral line (Vladykov,

496 1963; Vladykov and Gruchy, 1972). Indeed, the two genera are not closely allied in any of the

497 results presented here, nor has a close relationship of the two genera been reported in any other

498 molecular phylogenetic studies (e.g., Campbell et al., 2013; Crespi and Fulton, 2004; Matveev et

499 al., 2007; Oakley and Phillips, 1999). Though there has been considerable disagreement in the 
500 placement of Parahucho, the emerging consensus from nuclear gene sequences supports a

501 Parahucho + Salmo sister relationship (e.g., Lecaudey et al., 2018), as is recovered here.

502

503 Phylogenetic results from disomic and tetrasomic loci

504 Resolving subfamily relationships was a primary aim of this study, but our results repeatedly

505 showed a conflict in this segment of the phylogeny between analyses of the most stringently

506 assembled dataset (i.e., 100\% coverage) and of the remaining alignments, and particularly of the

507 tetrasomic loci. For example, in GT-ST analyses conducted with StarBEAST2 of the 100\%,

508 95\%, 93\% and 75\% coverage alignment Coregoninae and Thymallinae are supported as sister

509 lineages. Analyses of the 100\% coverage data set yield very strong (0.98 PP) support for that

510 grouping, which declines substantially in analyses of the other datasets $(0.34-0.52 \mathrm{PP})$. On the

511 other hand, the 33 tetrasomic loci we removed strongly supported a Coregoninae + Salmoninae

512 clade (0.99 PP). Likewise, we observed strong support for a sister relationship between Salmo

513 and Parahucho in the more reduced datasets (0.98-1.00 PP), and less support in the more

514 extensive $75 \%$ alignment (0.94 PP). The topology of the species tree inferred from StarBEAST2

515 analysis of tetrasomic loci indicates that Salmo and Parahucho are not closely related (Figure

516 4D). In summary, two of three sets of alternative placements we considered in detail (i.e.,

517 subfamily relationships, Coregonus monophyly, and placement of Parahucho) in this study yield

518 conflicting results when comparing datasets of loci with differing ploidy levels. We also find that

519 support values for the $\mathrm{C}+\mathrm{T}$ clade decline with greater proportions of missing data in the

520 underlying dataset.

521

522 We attempted to identify tetrasomic loci in the sequenced dataset through the assembly of a

523 single contig matching a UCE locus that was placed within genomic locations of known

524 tetrasomy in rainbow trout and Atlantic salmon. While there is evidence for broad conservation

525 of homologous tetrasomic genomic regions in salmonids (e.g., Brieuc et al., 2014; Kodama et al.,

526 2014; Lien et al., 2011; Sutherland et al., 2016), there is also evidence of substantial lineage-

527 specific rediploidization in salmonids and polyploids in general taxa (e.g., Robertson et al., 2017;

528 Spoelhof et al., 2017). Our definition of tetrasomic regions from two salmonines is

529 taxonomically restrictive and may have allowed the inclusion of regions of tetrasomic

530 inheritance in other species sequenced. Consequently, increasing the proportion of missing data 
531 may have introduced paralogous loci into our analyses due to lineage-specific rediploidization

532 processes (e.g., Figure 3B).

533

\section{CONCLUSION}

535 In salmonids, ancestral autopolyploidy and the resulting residual tetrasomy increases the 536 potential for conflicts between gene trees and species trees due to a greater likelihood for 537 incomplete lineage sorting. Lineage-specific rediploidization processes also are known to be 538 concentrated in the same tetrasomically pairing chromosome regions contributing to the analysis 539 of paralogous loci. Applying stringent criteria aimed at minimizing these problems yielded a

540 highly reduced dataset comprising six UCE loci. These loci show clear support for a

541 Coregoninae + Thymallinae clade and for the placement of Parahucho sister to Salmo, but do not 542 support the monophyly of Coregonus in joint GT and ST analysis. Expanding the number of

543 aligned loci by lowering stringency of filtering resulted in generally reduced confidence in clade

544 makeup and support for conflicting phylogenetic relationships while increasing support for the

545 monophyly of Coregonus. The use of tetrasomic loci likewise resulted in an alternative topology

546 from that of our $100 \%$ coverage analysis, and contradictory relationships may exhibit high

547 support values. The accurate inference of salmonid phylogenies is challenged not only by limits

548 of data and the sophistication of analyses, but also in part due to the evolutionary history and 549 patterns of the lineage.

550

\section{Acknowledgements}

552 We would like to thank Scott Edwards and Lang Liu for providing code and a manuscript in 553 advance of publication along with helpful advice for hypothesis testing under the multispecies

554 coalescent. We would also like to thank Max Belasco for his assistance generating the sequence 555 data used in this study. Michio Fukushima kindly allowed us to use a picture of Parahucho 556 perryi, Armando Piccinini and Stefano Porcellotti who allowed us to use a picture of Hucho 557 hucho, and Beatrice Smith who allowed us to use a picture of Stenodus leucichthys to create a 558 depiction of each of these species for our Figure 5. We would like to recognize Kevin 559 McCracken (University of Miami) for his support in promoting Next-Generation Sequencing and 560 Bioinformatics and the University of Alaska Fairbanks from which this data set was generated. 
561 Tissues in this study were provided by the University of Alaska Museum of the North, the Burke

562 Museum (University of Washington) and the Academy of Natural Sciences of Drexel University. 563

\section{Funding Statement}

565 This study was funded by a Genomics Seed Grant from the Alaska Idea Network for Biomedical 566 Research (INBRE) awarded to MAC and JAL. Research reported in this publication was

567 supported by an Institutional Development Award (IDeA) from the National Institute of General 568 Medical Sciences of the National Institutes of Health under grant number P20GM103395. The 569 content is solely the responsibility of the authors and does not necessarily reflect the official 570 views of the NIH. 


\section{Bibliography}

572 Alexandrou, M.A., Swartz, B.A., Matzke, N.J., Oakley, T.H., 2013. Genome duplication and

573

574

575

576

577

578

579

580

581

582

583

584

585

586

587

588

589

590

591

592

593

594

595

596

597

598

599

600

multiple evolutionary origins of complex migratory behavior in Salmonidae. Mol.

Phylogenet. Evol. 69, 514-523. https://doi.org/ 10.1016/j.ympev.2013.07.026

Allendorf, F.W., Thorgaard, G.H., 1984. Tetraploidy and the evolution of salmonid fishes. Evol. Genet. Fishes 1-53.

Altschul, S.F., Gish, W., Miller, W., Myers, E.W., Lipman, D.J., 1990. Basic local alignment search tool. J. Mol. Biol. 215, 403-410. https://doi.org/10.1016/S0022-2836(05)80360-2

Bernatchez, L., Colombani, F., Dodson, J.J., 1991a. Phylogenetic relationships among the subfamily Coregoninae as revealed by mitochondrial DNA restriction analysis. J. Fish Biol. 39, 283-290. https://doi.org/10.1111/j.1095-8649.1991.tb05091.x

Bernatchez, L., Edge, T.A., Dodson, J.J., Qadri, S.U., 1991b. Mitochondrial DNA and isozyme electrophoretic analyses of the endangered Acadian whitefish, Coregonus huntsmani Scott, 1987. Can. J. Zool. 69, 311-316. https://doi.org/10.1139/z91-050

Berthelot, C., Brunet, F., Chalopin, D., Juanchich, A., Bernard, M., Noel, B., Bento, P., Da Silva, C., Labadie, K., Alberti, A., Aury, J.-M., Louis, A., Dehais, P., Bardou, P., Montfort, J., Klopp, C., Cabau, C., Gaspin, C., Thorgaard, G.H., Boussaha, M., Quillet, E., Guyomard, R., Galiana, D., Bobe, J., Volff, J.-N., Genet, C., Wincker, P., Jaillon, O., Roest Crollius, H., Guiguen, Y., 2014. The rainbow trout genome provides novel insights into evolution after whole-genome duplication in vertebrates. Nat. Commun. 5, 3657. https://doi.org/10.1038/ncomms4657

Betancur-R, R., Broughton, R.E., Wiley, E.O., Carpenter, K., López, J.A., Li, C., Holcroft, N.I., Arcila, D., Sanciangco, M., Ii, J.C.C., Zhang, F., Buser, T., Campbell, M.A., Ballesteros, J.A., Roa-Varon, A., Willis, S., Borden, W.C., Rowley, T., Reneau, P.C., Hough, D.J., Lu, G., Grande, T., Arratia, G., Ortí, G., 2013. The Tree of Life and a New Classification of Bony Fishes. PLOS Curr. Tree Life. https://doi.org/10.1371/currents.tol.53ba26640df0ccaee75bb165c8c26288

Betancur-R, R., Wiley, E.O., Arratia, G., Acero, A., Bailly, N., Miya, M., Lecointre, G., Ortí, G., 2017. Phylogenetic classification of bony fishes. BMC Evol. Biol. 17, 162. https://doi.org/10.1186/s12862-017-0958-3 
601 Blumstein, D.M., Campbell, M.A., Hale, M.C., Sutherland, B.J.G., McKinney, G.J., Stott, W., 602 Larson, W.A., 2019. Comparative genomic analyses and a novel linkage map for cisco 603 (Coregonus artedi) provides insight into chromosomal evolution and rediploidization 604 across salmonids. bioRxiv 834937. https://doi.org/10.1101/834937

605 Bodaly, R.A., Vuorinen, J., Ward, R.D., Luczynski, M., Reist, J.D., 1991. Genetic comparisons 606 607 of New and Old World coregonid fishes. J. Fish Biol. 38, 37-51. https://doi.org/10.1111/j.1095-8649.1991.tb03089.x

Bolger, A.M., Lohse, M., Usadel, B., 2014. Trimmomatic: a flexible trimmer for Illumina sequence data. Bioinformatics 30, 2114-2120. https://doi.org/10.1093/bioinformatics/btu170

Bouckaert, R., Heled, J., Kühnert, D., Vaughan, T., Wu, C.-H., Xie, D., Suchard, M.A., Rambaut, A., Drummond, A.J., 2014. BEAST 2: a software platform for Bayesian evolutionary analysis. PLoS Comput. Biol. 10, e1003537. https://doi.org/10.1371/journal.pcbi.1003537

Brevoort, J.C., 1856. Notes on some figures of Japanese fish taken from recent specimens by the artists of the U. S. Japan Expedition, in: Narrative of the Expedition of an American Squadron to the China Seas and Japan, Performed in the Years 1852, 1853, and 1854 under the Command of Commodore M. C. Perry, United States Navy, by Order of the Government of the United States., U.S. Senate Ex. Doc. No. 79, 33rd Congress, 2nd Session. Beverley Tucker, Washington, D.C., pp. 253-288.

Brieuc, M.S.O., Waters, C.D., Seeb, J.E., Naish, K.A., 2014. A dense linkage map for Chinook salmon (Oncorhynchus tshawytscha) reveals variable chromosomal divergence after an ancestral whole genome duplication event. G3 GenesGenomesGenetics 4, 447. https://doi.org/10.1534/g3.113.009316

Burridge, C.P., McDowall, R.M., Craw, D., Wilson, M.V., Waters, J.M., 2012. Marine dispersal as a pre-requisite for Gondwanan vicariance among elements of the galaxiid fish fauna. J. Biogeogr. 39, 306-321. https://doi.org/10.1111/j.1365-2699.2011.02600.x

Campbell, M.A., Alfaro, M.E., Belasco, M., López, J.A., 2017. Early-branching euteleost relationships: areas of congruence between concatenation and coalescent model inferences. PeerJ 5, e3548. https://doi.org/10.7717/peerj.3548 
631 Campbell, M.A., Chen, W.-J., López, J.A., 2014. Molecular data do not provide unambiguous

632

633

634

635

636

637

638

639

640

641

642

643

644

645

646

647

648

649

650

651

652

653

654

655

656

657

658

659

660 support for the monophyly of flatfishes (Pleuronectiformes): A reply to Betancur-R and Ortí. Mol. Phylogenet. Evol. 75, 149-153. https://doi.org/10.1016/j.ympev.2014.02.011

Campbell, M.A., Ganley, A.R.D., Gabaldón, T., Cox, M.P., 2016. The case of the missing ancient fungal polyploids. Am. Nat. 188, 602-614. https://doi.org/10.1086/688763

Campbell, M.A., Hale, M.C., McKinney, G.J., Nichols, K.M., Pearse, D.E., 2019. Long-term conservation of ohnologs through partial tetrasomy following whole-genome duplication in Salmonidae. G3 GenesGenomesGenetics 9, 2017. https://doi.org/10.1534/g3.119.400070

Campbell, M.A., López, J.A., Sado, T., Miya, M., 2013. Pike and salmon as sister taxa: Detailed intraclade resolution and divergence time estimation of Esociformes + Salmoniformes based on whole mitochondrial genome sequences. Gene 530, 57-65. https://doi.org/10.1016/j.gene.2013.07.068

Campbell, Matthew.A., Sado, T., Shinzato, C., Koyanagi, R., Okamoto, M., Miya, M., 2018. Multi-locus phylogenetic analysis of the first molecular data from the rare and monotypic Amarsipidae places the family within the Pelagia and highlights limitations of existing data sets in resolving pelagian interrelationships. Mol. Phylogenet. Evol. 172-180. https://doi.org/10.1016/j.ympev.2005.10.007

Cranston, K., 2010. Summarizing gene tree incongruence at multiple phylogenetic depths, in: Knowles, L.L., Kubatko, L.S. (Eds.), Estimating Species Trees: Practical and Theoretical Aspects. John Wiley \& Sons, Inc, Hoboken, New Jersey, pp. 129-142.

Crespi, B.J., Fulton, M.J., 2004. Molecular systematics of Salmonidae: combined nuclear data yields a robust phylogeny. Mol. Phylogenet. Evol. 31, 658-679. https://doi.org/10.1016/j.ympev.2003.08.012

Crête-Lafrenière, A., Weir, L.K., Bernatchez, L., 2012. Framing the Salmonidae family phylogenetic portrait: a more complete picture from increased taxon sampling. PLoS One 7, e46662. https://doi.org/10.1371/journal.pone.0046662

Degnan, J.H., Rosenberg, N.A., 2009. Gene tree discordance, phylogenetic inference and the multispecies coalescent. Trends Ecol. Evol. 24, 332-340. https://doi.org/10.1016/j.tree.2009.01.009 
661 Dehal, P., Boore, J.L., 2005. Two rounds of whole genome duplication in the ancestral

662

663

664

665

666

667

668

669

670

671

672

673

674

675

676

677

678

679

680

681

682

683

684

685

686

687

688

689

690

691 vertebrate. PLoS Biol. 3, e314. https://doi.org/10.1371/journal.pbio.0030314

Faircloth, B.C., 2015. PHYLUCE is a software package for the analysis of conserved genomic loci. Bioinformatics. https://doi.org/10.1093/bioinformatics/btv646

Faircloth, B.C., Sorenson, L., Santini, F., Alfaro, M.E., 2013. A phylogenomic perspective on the radiation of ray-finned fishes based upon targeted sequencing of Ultraconserved Elements (UCEs). PLoS One 8. https://doi.org/10.1371/journal.pone.0065923

Fricke, R., Eschmeyer, W., Fong, J.D., 2019. Catalog of Fishes. http://researcharchive.calacademy.org/research/ichthyology/catalog/SpeciesByFamily.asp

Friedman, M., Feilich, K.L., Beckett, H.T., Alfaro, M.E., Faircloth, B.C., Černý, D., Miya, M., Near, T.J., Harrington, R.C., 2019. A phylogenomic framework for pelagiarian fishes (Acanthomorpha: Percomorpha) highlights mosaic radiation in the open ocean. Proc. R. Soc. B Biol. Sci. 286, 20191502. https://doi.org/10.1098/rspb.2019.1502

Fukushima, M., Shimazaki, H., Rand, P.S., Kaeriyama, M., 2011. Reconstructing Sakhalin taimen Parahucho perryi historical distribution and identifying causes for local extinctions. Trans. Am. Fish. Soc. 140, 1-13. https://doi.org/10.1080/00028487.2011.544999

Gerstein, A.C., Otto, S.P., 2009. Ploidy and the causes of genomic evolution. J. Hered. 100, 571581. https://doi.org/10.1093/jhered/esp057

Gilbert, P.S., Chang, J., Pan, C., Sobel, E.M., Sinsheimer, J.S., Faircloth, B.C., Alfaro, M.E., 2015. Genome-wide ultraconserved elements exhibit higher phylogenetic informativeness than traditional gene markers in percomorph fishes. Mol. Phylogenet. Evol. 92, 140-146. https://doi.org/10.1016/j.ympev.2015.05.027

Gladman, S., Seemann, T., 2012. VelvetOptimiser. https:/github.com/Victorian-BioinformaticsConsortium/VelvetOptimiser

Günther, A.C.L.G., 1868. Catalogue of the Fishes in the British Museum. Catalogue of the Physostomi, containing the families Salmonidae, Percopsidae, Galaxidae, Mormyridae, Gymnarchidae, Esocidae, Umbridae, Scombresocidae, Cyprinodontidae, in the collection of the British Museum. British Musesum of Natural History.

Horreo, J.L., 2017. Revisiting the mitogenomic phylogeny of Salmoninae: new insights thanks to recent sequencing advances. PeerJ 5, e3828. https://doi.org/10.7717/peerj.3828 
692 Ishiguro, N.B., Miya, M., Nishida, M., 2003. Basal euteleostean relationships: a mitogenomic

693

694

695

696

697

698

699

700

701

702

703

704

705

706

707

708

709

710

711

712

713

714

715

716

717

718

719

720

721 perspective on the phylogenetic reality of the "Protacanthopterygii." Mol. Phylogenet. Evol. 27, 476-488. https://doi.org/doi: DOI: 10.1016/S1055-7903(02)00418-9

Jeffreys, H., 1935. Some tests of significance, treated by the theory of probability, in: Mathematical Proceedings of the Cambridge Philosophical Society. Cambridge University Press, pp. 203-222.

Jordan, D.S., Snyder, J.O., 1902. A review of the salmonoid fishes of Japan. Proc. U. S. Natl. Mus.

Kass, R.E., Raftery, A.E., 1995. Bayes factors. J. Am. Stat. Assoc. 90, 773-795.

Katoh, K., Misawa, K., Kuma, K., Miyata, T., 2002. MAFFT: a novel method for rapid multiple sequence alignment based on fast Fourier transform. Nucleic Acids Res. 30, 3059-3066. https://doi.org/10.1093/nar/gkf436

Katoh, K., Standley, D.M., 2013. MAFFT multiple sequence alignment software version 7: improvements in performance and usability. Mol. Biol. Evol. 30, 772-780. https://doi.org/10.1093/molbev/mst010

Katoh, K., Toh, H., 2008. Recent developments in the MAFFT multiple sequence alignment program. Brief. Bioinform. 9, 286-298. https://doi.org/10.1093/bib/bbn013

Keane, T.M., Creevey, C.J., Pentony, M.M., Naughton, T.J., Mclnerney, J.O., 2006. Assessment of methods for amino acid matrix selection and their use on empirical data shows that ad hoc assumptions for choice of matrix are not justified. BMC Evol. Biol. 6, 29. https://doi.org/10.1186/1471-2148-6-29

Kendall Jr., A.W., Behnke, R.J., 1984. Salmonidae: Development and relationships., in: Moser, H.G., Richards, W.J., Cohen, D.M., Fahay, M.P., Kendall Jr., A.W., Richards, S.L. (Eds.), Ontogeny and Sytematics of Fishes, Special Publication. American Society of Ichthyologists and Herpetologists, pp. 142-149.

Kimura, S., 1966. On the life history of the salmonid fish, Hucho perryi (Brevoort), found in Nemuro, Hokkaido. Jpn. J. Ichthyol. 14, 17-25_2.

Kodama, M., Brieuc, M.S.O., Devlin, R.H., Hard, J.J., Naish, K.A., 2014. Comparative mapping between coho Salmon (Oncorhynchus kisutch) and three other salmonids suggests a role for chromosomal rearrangements in the retention of duplicated regions following a whole 
722

723

724

725

726

727

728

729

730

731

732

733

734

735

736

737

738

739

740

741

742

743

744

745

746

747

748

749

750

751

genome duplication event. G3 GenesGenomesGenetics 4, 1717.

https://doi.org/10.1534/g3.114.012294

Lanfear, R., Calcott, B., Ho, S.Y.W., Guindon, S., 2012. PartitionFinder: combined selection of partitioning schemes and substitution models for phylogenetic analyses. Mol. Biol. Evol. 29, 1695-1701. https://doi.org/10.1093/molbev/mss020

Lanfear, R., Calcott, B., Kainer, D., Mayer, C., Stamatakis, A., 2014. Selecting optimal partitioning schemes for phylogenomic datasets. BMC Evol. Biol. 14, 1-14. https://doi.org/10.1186/1471-2148-14-82

Lecaudey, L.A., Schliewen, U.K., Osinov, A.G., Taylor, E.B., Bernatchez, L., Weiss, S.J., 2018. Inferring phylogenetic structure, hybridization and divergence times within Salmoninae (Teleostei: Salmonidae) using RAD-sequencing. Mol. Phylogenet. Evol. 124, 82-99. https://doi.org/10.1016/j.ympev.2018.02.022

Leggatt, RosalindA., Iwama, GeorgeK., 2003. Occurrence of polyploidy in the fishes. Rev. Fish Biol. Fish. 13, 237-246. https://doi.org/10.1023/B:RFBF.0000033049.00668.fe

Li, J., Xia, R., McDowall, R.M., López, J.A., Lei, G., Fu, C., 2010. Phylogenetic position of the enigmatic Lepidogalaxias salamandroides with comment on the orders of lower euteleostean fishes. Mol. Phylogenet. Evol. 57, 932-936. https://doi.org/10.1016/j.ympev.2010.07.016

Lien, S., Gidskehaug, L., Moen, T., Hayes, B.J., Berg, P.R., Davidson, W.S., Omholt, S.W., Kent, M.P., 2011. A dense SNP-based linkage map for Atlantic salmon (Salmo salar) reveals extended chromosome homeologies and striking differences in sex-specific recombination patterns. BMC Genomics 12, 615. https://doi.org/10.1186/1471-2164-12615

Lien, S., Koop, B.F., Sandve, S.R., Miller, J.R., Kent, M.P., Nome, T., Hvidsten, T.R., Leong, J.S., Minkley, D.R., Zimin, A., Grammes, F., Grove, H., Gjuvsland, A., Walenz, B., Hermansen, R.A., von Schalburg, K., Rondeau, E.B., Di Genova, A., Samy, J.K.A., Olav Vik, J., Vigeland, M.D., Caler, L., Grimholt, U., Jentoft, S., Inge Våge, D., de Jong, P., Moen, T., Baranski, M., Palti, Y., Smith, D.R., Yorke, J.A., Nederbragt, A.J., ToomingKlunderud, A., Jakobsen, K.S., Jiang, X., Fan, D., Hu, Y., Liberles, D.A., Vidal, R., Iturra, P., Jones, S.J.M., Jonassen, I., Maass, A., Omholt, S.W., Davidson, W.S., 2016. 
752

753

754

755

756

757

758

759

760

761

762

763

764

765

766

767

768

769

770

771

772

773

774

775

776

777

778

779

780

781

782

The Atlantic salmon genome provides insights into rediploidization. Nature 533, 200 205. https://doi.org/10.1038/nature17164

Liu, L., Anderson, C., Pearl, D., Edwards, S.V., 2019. Modern phylogenomics: building phylogenetic trees using the multispecies coalescent model, in: Anisimova, M. (Ed.), Evolutionary Genomics: Statistical and Computational Methods. Springer.

Liu, L., Yu, L., 2010. Phybase: an R package for species tree analysis. Bioinformatics 26, 962963. https://doi.org/10.1093/bioinformatics/btq062

Liu, L., Yu, L., Edwards, S.V., 2010. A maximum pseudo-likelihood approach for estimating species trees under the coalescent model. BMC Evol. Biol. 10, 302. https://doi.org/10.1186/1471-2148-10-302

López, J.A., Chen, W.J., Ortí, G., 2004. Esociform phylogeny. Copeia 2004, 449-464. https://doi.org/doi: 10.1643/CG-03-087R1

Macqueen, D.J., Johnston, I.A., 2014. A well-constrained estimate for the timing of the salmonid whole genome duplication reveals major decoupling from species diversification. Proc. R. Soc. B Biol. Sci. 281. https://doi.org/10.1098/rspb.2013.2881

Mallet, J., 2005. Hybridization as an invasion of the genome. Trends Ecol. Evol. 20, 229-237. https://doi.org/10.1016/j.tree.2005.02.010

Mallo, D., Posada, D., 2016. Multilocus inference of species trees and DNA barcoding. Philos. Trans. R. Soc. Lond. B. Biol. Sci. 371, 20150335. https://doi.org/10.1098/rstb.2015.0335

Matveev, V., Nishihara, H., Okada, N., 2007. Novel SINE families from salmons validate Parahucho (Salmonidae) as a distinct genus and give evidence that SINEs can incorporate LINE-related 3'-tails of other SINEs. Mol. Biol. Evol. 24, 1656-1666.

Meyer, A., Van de Peer, Y., 2005. From 2R to 3R: evidence for a fish-specific genome duplication (FSGD). BioEssays 27, 937-945. https://doi.org/10.1002/bies.20293

Meyers, L.A., Levin, D.A., Geber, M., 2006. On the abundance of polyploids in flowering plants. Evolution 60, 1198-1206. https://doi.org/10.1554/05-629.1

Miya, M., Friedman, M., Satoh, T.P., Takeshima, H., Sado, T., Iwasaki, W., Yamanoue, Y., Nakatani, M., Mabuchi, K., Inoue, J.G., Poulsen, J.Y., Fukunaga, T., Sato, Y., Nishida, M., 2013. Evolutionary origin of the Scombridae (Tunas and Mackerels): Members of a Paleogene adaptive radiation with 14 other pelagic fish families. PLoS ONE 8, e73535. https://doi.org/10.1371/journal.pone.0073535 
783 Muir, P., Li, S., Lou, S., Wang, D., Spakowicz, D.J., Salichos, L., Zhang, J., Weinstock, G.M.,

784

785

786

787

788

789

790

791

792

793

794

795

796

797

798

799

800

801

802

803

804

805

806

807

808

809

810

811

812

Isaacs, F., Rozowsky, J., Gerstein, M., 2016. The real cost of sequencing: scaling computation to keep pace with data generation. Genome Biol. 17, 53. https://doi.org/10.1186/s13059-016-0917-0

Near, T.J., Eytan, R.I., Dornburg, A., Kuhn, K.L., Moore, J.A., Davis, M.P., Wainwright, P.C., Friedman, M., Smith, W.L., 2012. Resolution of ray-finned fish phylogeny and timing of diversification. Proc. Natl. Acad. Sci. 109, 13698-13703.

Nelson, J.S., 2006. Fishes of the world, 4th ed. John Wiley and Sons, Inc., New York.

Norden, C.R., 1961. Comparative osteology of representative salmonid fishes, with particular reference to the grayling (Thymallus arcticus) and its phylogeny. J. Fish. Board Can. 18, 679-791.

Oakley, T.H., Phillips, R.B., 1999. Phylogeny of salmonine fishes based on growth hormone introns: Atlantic (Salmo) and Pacific (Oncorhynchus) salmon are not sister taxa. Mol. Phylogenet. Evol. 11, 381-393.

Ogilvie, H.A., Bouckaert, R.R., Drummond, A.J., 2017. StarBEAST2 rings faster species tree inference and accurate estimates of substitution rates. Mol. Biol. Evol. 34, 2101-2114. https://doi.org/10.1093/molbev/msx126

Ohno, S., 1970. Evolution by gene duplication. New York: Springer-Verlag.

Pamilo, P., Nei, M., 1988. Relationships between gene trees and species trees. Mol. Biol. Evol. $5,568-583$.

Pearse, D.E., Barson, N.J., Nome, T., Gao, G., Campbell, M.A., Abadía-Cardoso, A., Anderson, E.C., Rundio, D.E., Williams, T.H., Naish, K.A., Moen, T., Liu, S., Kent, M., Moser, M., Minkley, D.R., Rondeau, E.B., Brieuc, M.S.O., Sandve, S.R., Miller, M.R., Cedillo, L., Baruch, K., Hernandez, A.G., Ben-Zvi, G., Shem-Tov, D., Barad, O., Kuzishchin, K., Garza, J.C., Lindley, S.T., Koop, B.F., Thorgaard, G.H., Palti, Y., Lien, S., 2019. Sexdependent dominance maintains migration supergene in rainbow trout. Nat. Ecol. Evol. https://doi.org/10.1038/s41559-019-1044-6

Rambaut, A., Drummond, A.J., Xie, D., Baele, G., Suchard, M.A., 2018. Posterior summarization in Bayesian phylogenetics using Tracer 1.7. Syst. Biol. 67, 901-904. https://doi.org/10.1093/sysbio/syy032 
813 Rand, P.S., 2006. Hucho perryi. The IUCN Red List of Threatened Species:

814

e.T61333A12462795.

815

816

817

818

819

820

821

822

823

824

825

826

827

828

829

830

831

832

833

834

835

836

837

838

839

840

841

842

843 1241-z Zool. 56, 145-153.

Robertson, F.M., Gundappa, M.K., Grammes, F., Hvidsten, T.R., Redmond, A.K., Lien, S., Martin, S.A.M., Holland, P.W.H., Sandve, S.R., Macqueen, D.J., 2017. Lineage-specific rediploidization is a mechanism to explain time-lags between genome duplication and evolutionary diversification. Genome Biol. 18, 111. https://doi.org/10.1186/s13059-017-

Sajdak, S.L., Phillips, R.B., 1997. Phylogenetic relationships among Coregonus species inferred from the DNA sequence of the first internal transcribed spacer (ITS1) of ribosomal DNA. Can. J. Fish. Aquat. Sci. 54, 1494-1503. https://doi.org/10.1139/f97-057

Sanford, C.J., 1990. The phylogenetic relationships of salmonoid fishes. Bull. Br. Mus. Nat. Hist.

Scannell, D.R., Frank, A.C., Conant, G.C., Byrne, K.P., Woolfit, M., Wolfe, K.H., 2007. Independent sorting-out of thousands of duplicated gene pairs in two yeast species descended from a whole-genome duplication. Proc. Natl. Acad. Sci. 104, 8397-8402. https://doi.org/10.1073/pnas.0608218104

Shedko, S.V., Miroshnichenko, I.L., Nemkova, G.A., 2012. Phylogeny of salmonids (Salmoniformes: Salmonidae) and its molecular dating: Analysis of nuclear RAG1 gene. Russ. J. Genet. 48, 575-579.

Shimodaira, H., Hasegawa, M., 2001. CONSEL: for assessing the confidence of phylogenetic tree selection. Bioinformatics 17, 1246-1247. https://doi.org/10.1093/bioinformatics/17.12.1246

Smith, W.L., Elizabeth Everman, Clara Richardson, 2018. Phylogeny and Taxonomy of Flatheads, Scorpionfishes, Sea Robins, and Stonefishes (Percomorpha: Scorpaeniformes) and the Evolution of the Lachrymal Saber. Copeia 106, 94-119. https://doi.org/10.1643/CG-17-669

Spoelhof, J.P., Soltis, P.S., Soltis, D.E., 2017. Pure polyploidy: Closing the gaps in autopolyploid research. J. Syst. Evol. 55, 340-352. https://doi.org/10.1111/jse.12253

Stamatakis, A., 2014. RAxML version 8: a tool for phylogenetic analysis and post-analysis of large phylogenies. Bioinformatics 30, 1312-1313. https://doi.org/10.1093/bioinformatics/btu033 
844 Stearley, R.F., Smith, G.R., 1993. Phylogeny of the Pacific trouts and salmons (Oncorhynchus)

845

846

847

848

849

850

851

852

853

854

855

856

857

858

859

860

861

862

863

864

865

866

867

868

869

870

871

872

873

874

and genera of the family Salmonidae. Trans. Am. Fish. Soc. 122, 1-33. https://doi.org/10.1577/1548-8659

Sutherland, B.J.G., Gosselin, T., Normandeau, E., Lamothe, M., Isabel, N., Audet, C., Bernatchez, L., 2016. Salmonid chromosome evolution as revealed by a novel method for comparing RADseq linkage maps. Genome Biol. Evol. 8, 3600-3617. https://doi.org/10.1093/gbe/evw262

Vladykov, V.D., 1963. Review of salmonid genera and their broad geographical distribution. Trans. R. Soc. Can. 1, 459-.

Vladykov, V.D., Gruchy, C.G., 1972. Comments on the nomenclature of some subgenera of Salmonidae. J. Fish. Res. Board Can. 29, 1631-1632.

Vuorinen, J.A., Bodaly, R.A., Reist, J.D., Luczynski, M., 1998. Phylogeny of five Prosopium species with comparisons with other Coregonine fishes based on isozyme electrophoresis. J. Fish Biol. 53, 917-927.

Wendel, J.F., 2015. The wondrous cycles of polyploidy in plants. Am. J. Bot. 102, 1753-1756. https://doi.org/10.3732/ajb.1500320

Wilson, M.V.H., Li, G.-Q., 1999. Osteology and systematic position of the Eocene salmonid $\dagger$ Eosalmo driftwoodensis Wilson from western North America. Zool. J. Linn. Soc. 125, 279-311. https://doi.org/10.1111/j.1096-3642.1999.tb00594.x

Wilson, M.V.H., Williams, R.R.G., 2010. Salmoniform fishes: key fossils, supertree, and possible morphological synapomorphies, in: Nelson JS, Schultze HP, Wilson MVH, Editors. Origin and Phylogenetic Interrelationships of Teleosts. Verlag Dr. Friedrich Pfeil, München, pp. 379-409.

Yasuike, M., Jantzen, S., Cooper, G.A., Leder, E., Davidson, W.S., Koop, B.F., 2010. Grayling (Thymallinae) phylogeny within salmonids: complete mitochondrial DNA sequences of Thymallus arcticus and Thymallus thymallus. J. Fish Biol. 76, 395-400.

Yu, Y., Than, C., Degnan, J.H., Nakhleh, L., 2011. Coalescent histories on phylogenetic networks and detection of hybridization despite incomplete lineage sorting. Syst. Biol. 60, 138-149. https://doi.org/10.1093/sysbio/syq084

Zerbino, D.R., Birney, E., 2008. Velvet: Algorithms for de novo short read assembly using de Bruijn graphs. Genome Res. 18, 821-829. https://doi.org/10.1101/gr.074492.107 
Figure 1

Depiction of Gene Tree and Species Tree conflict.

(A) Hypothetical species relationships between three species, I, II and III. (B) Depiction of three possible gene trees. (C) Combined species tree and gene trees. Only one of the gene trees (blue) follows the species tree relationships. 
A. Species Relationships: ((I, II), III)

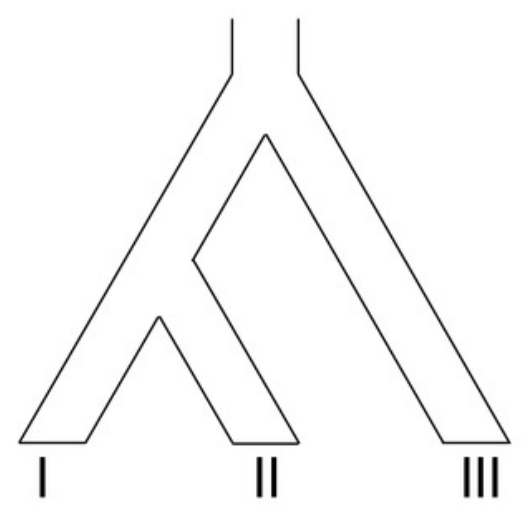

B. Possible Gene Trees

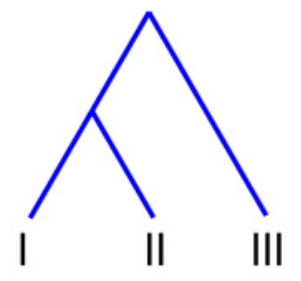

C. Different Gene Trees within Species Tree

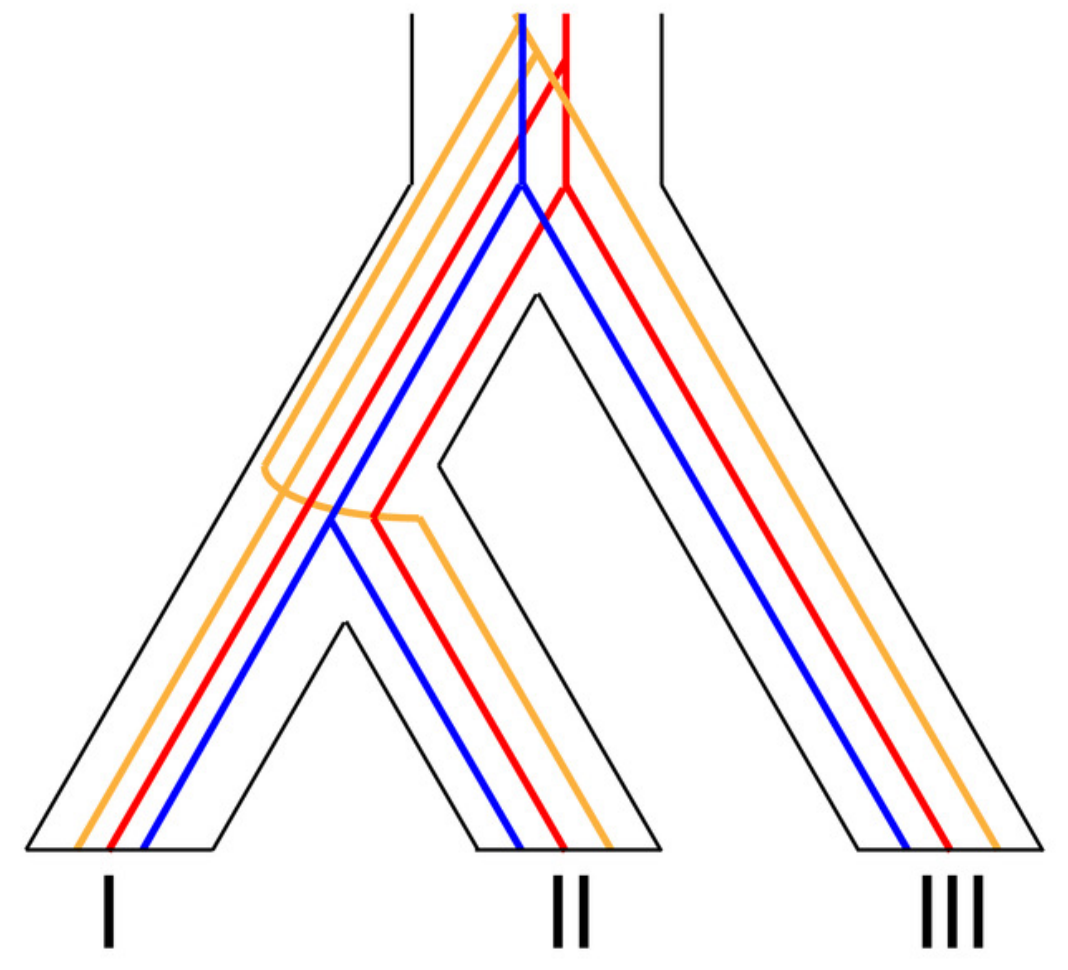




\section{Figure 2}

Effects of Time and Effective Population Size $\left(\mathrm{N}_{\mathrm{E}}\right)$ on Incomplete Lineage Sorting (ILS).

(A) Species tree depicting relationships between species I, II and III. The branch leading to the common ancestor of I \& II is indicated in a box as where the ratio of time (t, in

generations) versus $\mathrm{N}_{\mathrm{E}}$ is considered for the other subpanels. (B) Depiction of how increased population sizes increase ILS. A single coalescent event is depicted for each generation with population sizes of eight and four. (C) Depiction of decreased time increasing ILS. For a population of eight, four or eight generations are shown. (D) For a three-taxon species tree (as in Subpanel A), the probability of inferring a correct or incorrect gene tree is plotted as a function of $t / \mathrm{N}_{\mathrm{E}}$. As there are three possible outcomes, and only one correct one, the initial probability of inferring an incorrect gene tree is $2 / 3$ at $t / N_{E}=0$. The effect of different ploidy levels is shown, with increased ploidy increasing ILS. 


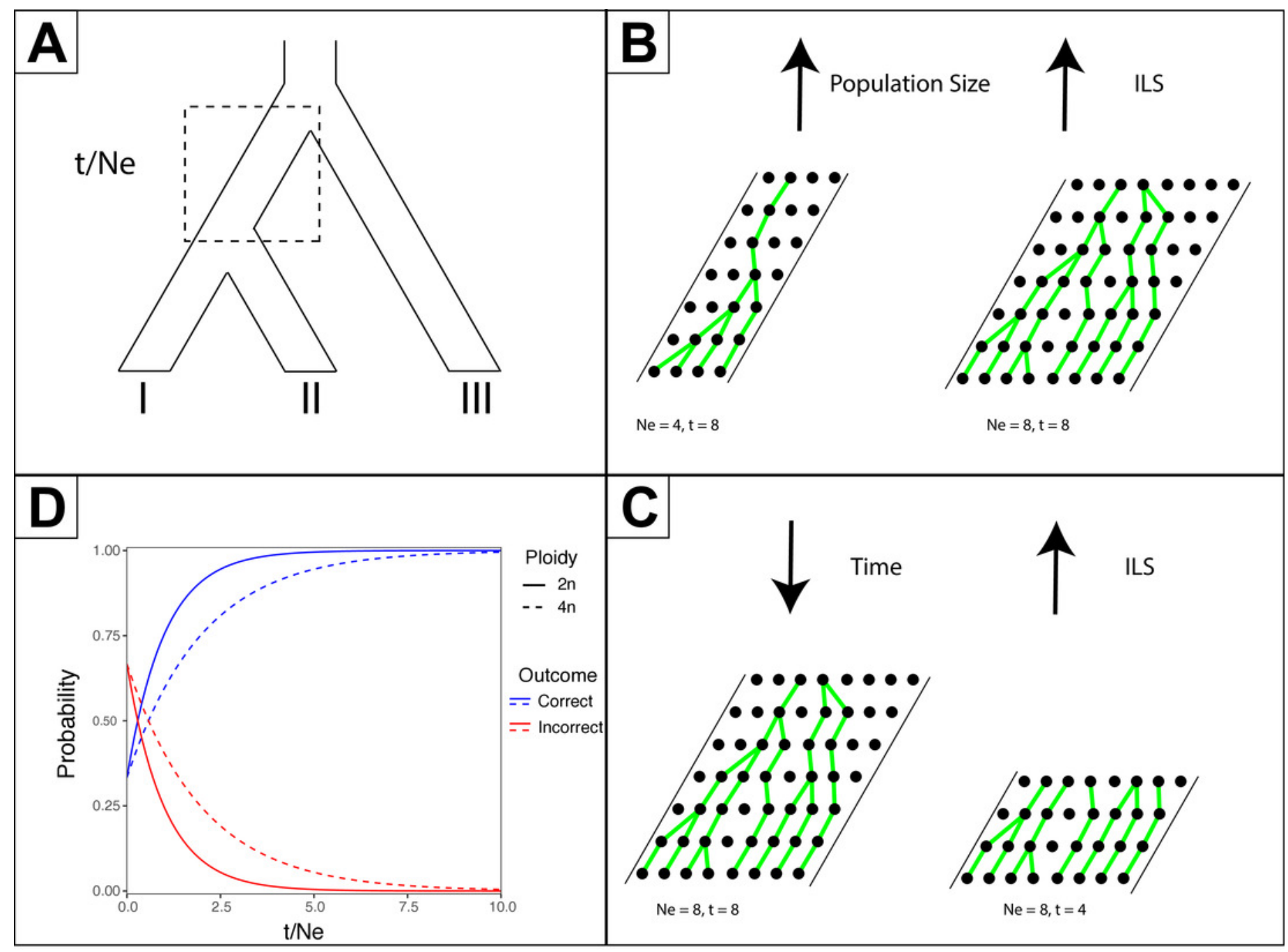




\section{Figure 3}

Example of Gene Duplication and Loss Misleading Phylogenetic Inference.

Species tree of three species depicting a gene duplication event in the common ancestor of all three species. (B) Example of loss of paralogs in different lineages. Each species exhibits a single copy of the formerly duplicated gene; however, construction of a phylogeny from those sequences would make it appear that species I and species III would be most closely related to each other.

A. Species Relationships: ((I, II), III)

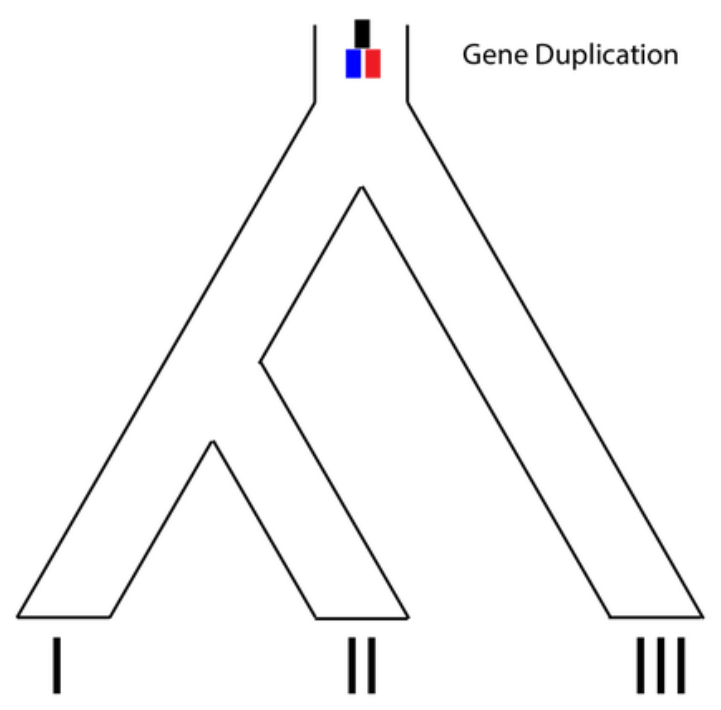

B. Duplication Followed by Loss

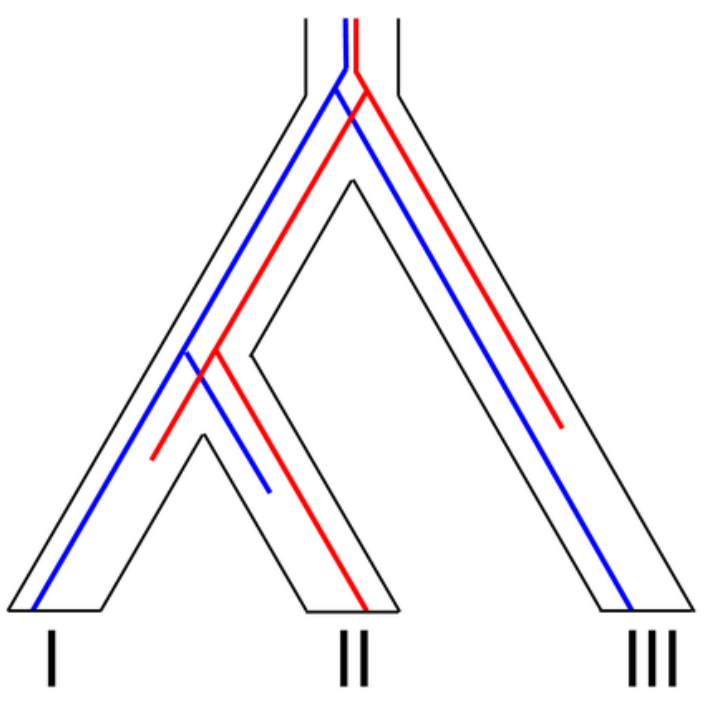

I \& III would appear most closely related to each other 
Figure 4

\section{Species Trees Generated in StarBEAST2.}

A joint estimation of species trees and gene trees is presented with posterior probability presented at nodes for four separate alignments. Posterior probabilities equal to 1.00 are not shown. (A) 100\% coverage alignment. (B) 95\% coverage alignment. (C) 93\% coverage alignment. (D) Tetrasomic loci alignment.

A. $100 \%$ Coverage Alignment

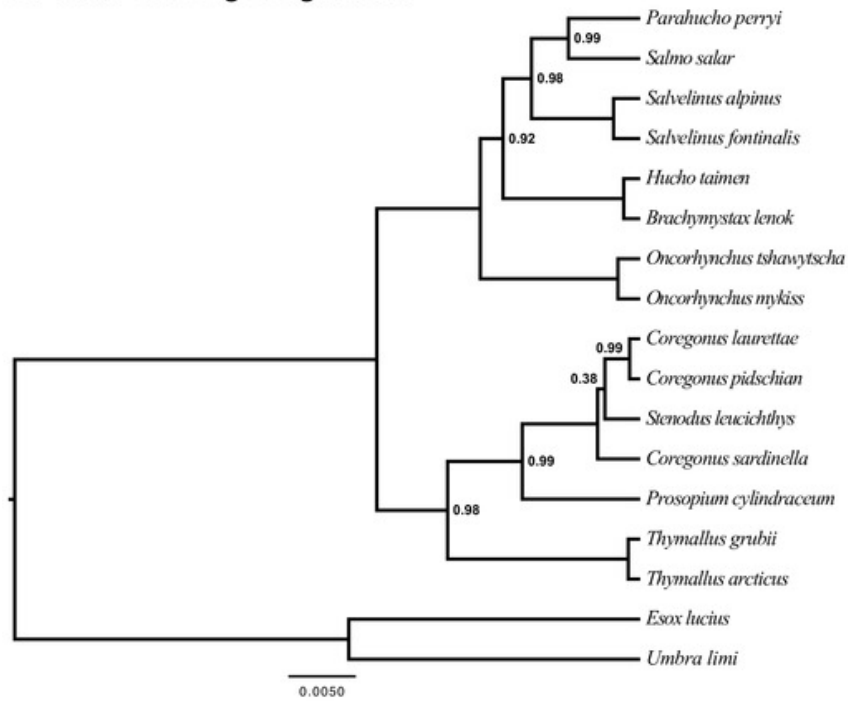

C. $93 \%$ Coverage Alignment

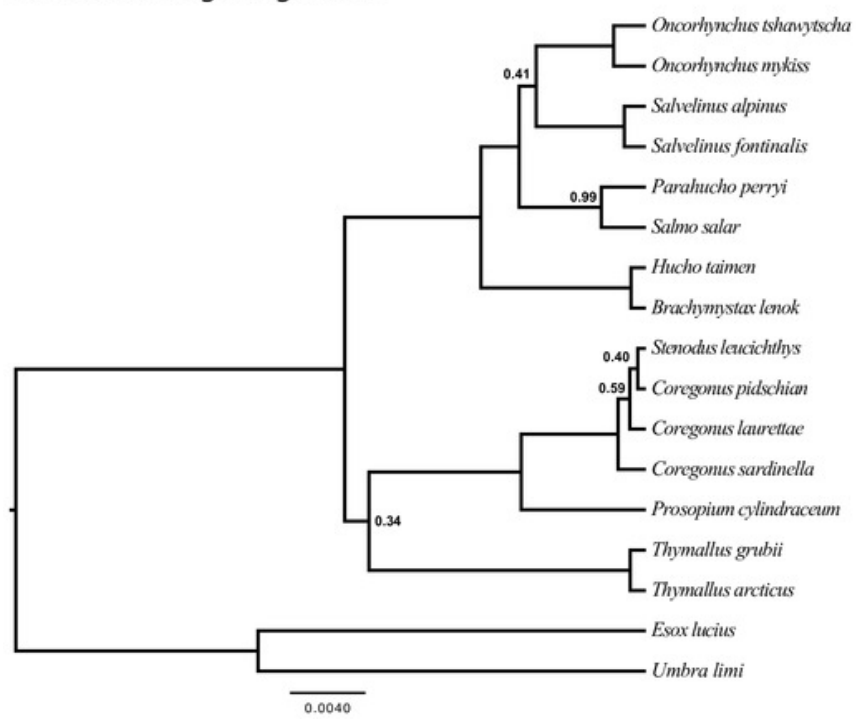

B. $95 \%$ Coverage Alignment

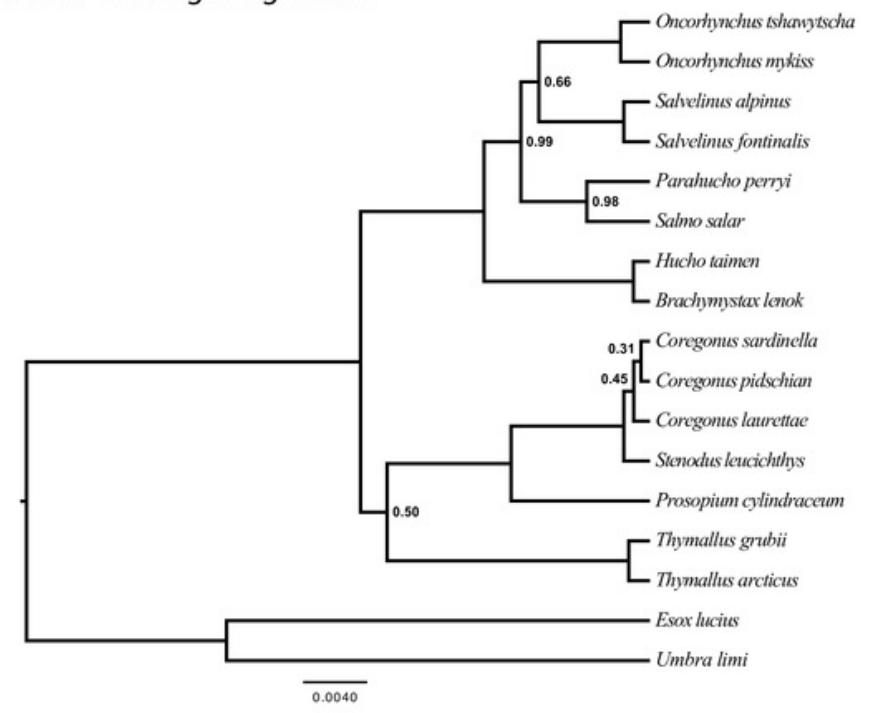

D. Tetrasomic Loci Alignment

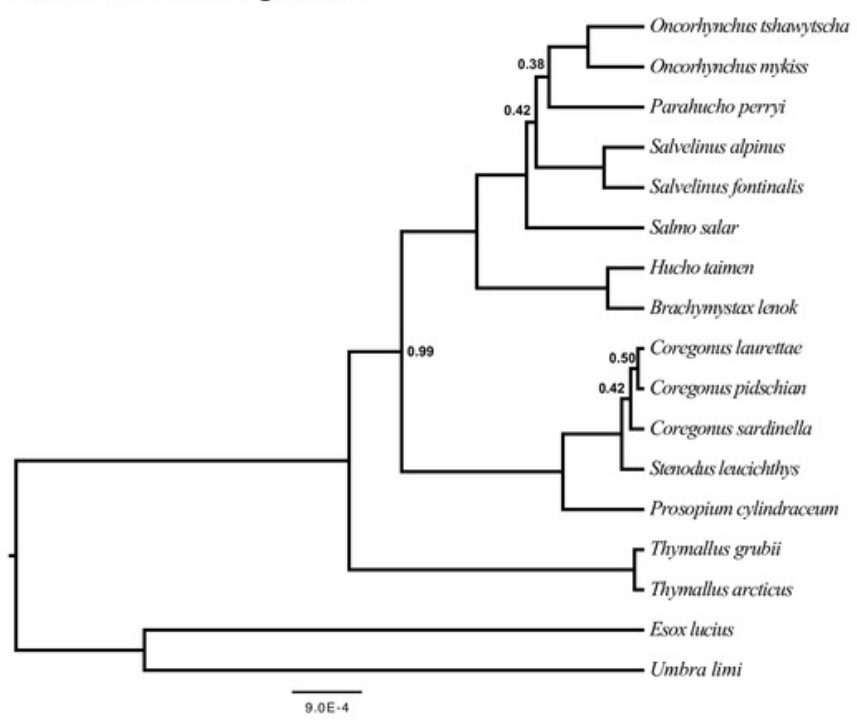


Figure 5

Species Tree Generated in StarBEAST2 from 75\% Coverage Alignment.

A joint estimation of species trees and gene trees is presented with posterior probability presented at nodes. Depictions of species in the phylogeny are presented with bold species names indicating the represented species. Hucho taimen is represented by a drawing of Hucho hucho. Drawings by Thaddaeus Buser.

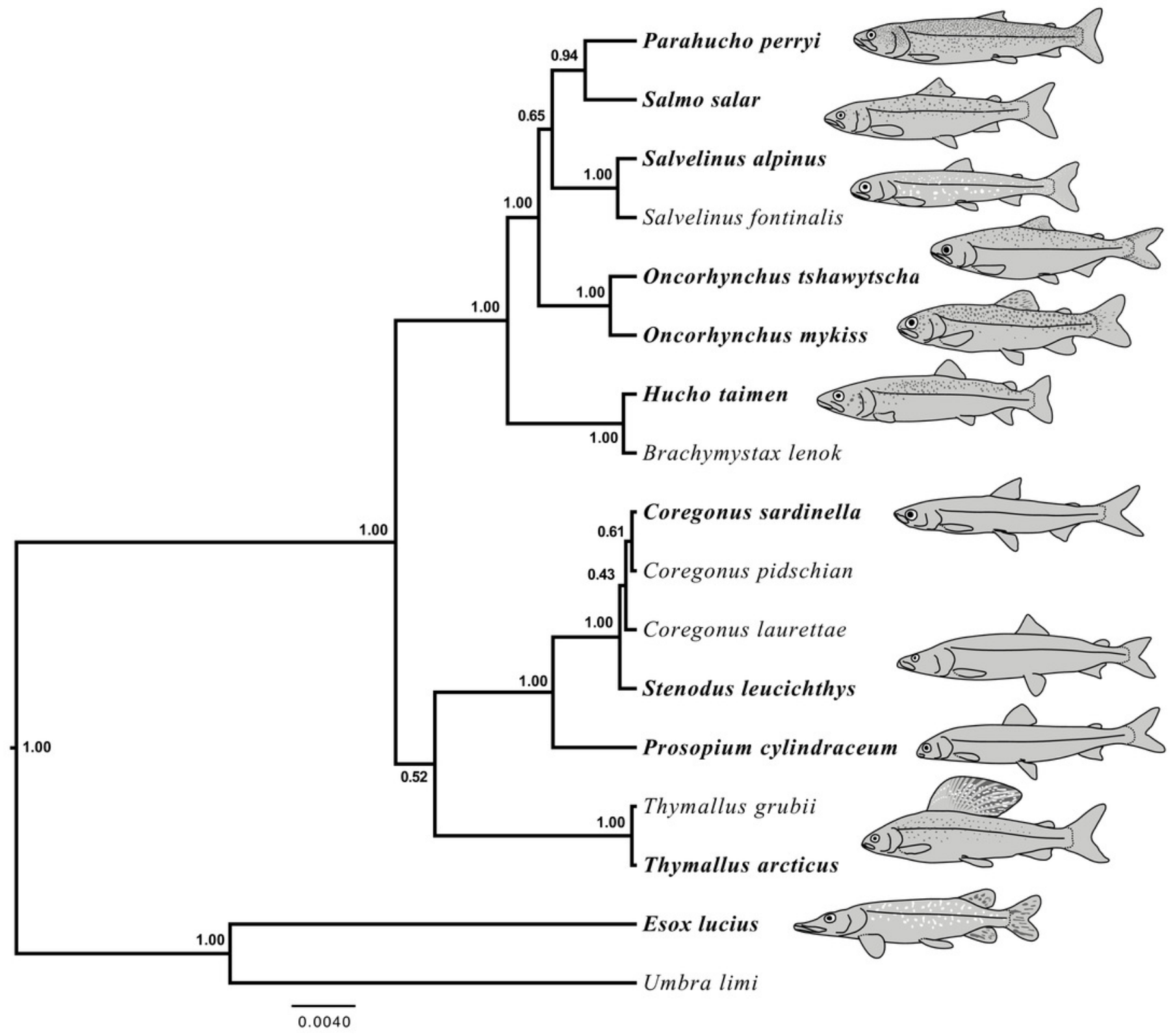




\section{Table $\mathbf{1}$ (on next page)}

Characteristics of UCE Alignments.

For the five alignments generated for the study, the amount of missing data allowed in terms of taxa/locus is given, the number of resulting UCEs generated, and the number that were considered tetrasomic are presented. The resulting alignment length, unique alignment patterns and percentage of gaps and undetermined portions are then provided. 


\begin{tabular}{|c|c|c|c|c|c|c|c|}
\hline Alignment Name & Missing Data Threshold & Number of UCEs & $\begin{array}{c}\text { Number of } \\
\text { Tetrasomic } \\
\text { Loci }\end{array}$ & $\begin{array}{c}\text { Number of } \\
\text { Loci Analyzed }\end{array}$ & $\begin{array}{c}\text { Alignment } \\
\text { Length }\end{array}$ & $\begin{array}{c}\text { Unique } \\
\text { Alignment } \\
\text { Patterns } \\
\end{array}$ & $\begin{array}{c}\text { Gaps and } \\
\text { Undetermined }\end{array}$ \\
\hline $100 \%$ Coverage & No missing taxa/locus & 8 & 2 & 6 & 2,331 & 645 & $15.63 \%$ \\
\hline $95 \%$ Coverage & 1 taxon/locus & 19 & 6 & 13 & 5,297 & 1,261 & $21.18 \%$ \\
\hline $93 \%$ Coverage & 2 taxa/locus & 42 & 13 & 28 & 10,232 & 2,382 & $21.89 \%$ \\
\hline $75 \%$ Coverage & 5 taxa/locus & 138 & 33 & 105 & 36,835 & 8,688 & $33.83 \%$ \\
\hline Tetrasomic loci & 5 taxa/locus & 138 & 33 & 33 & 12,758 & 2,661 & $33.13 \%$ \\
\hline
\end{tabular}




\section{Table 2 (on next page)}

Outcomes of Phylogenetic Analyses Incorporating the Multispecies Coalescent.

For the three methods employing the multispecies coalescent, StarBEAST2, ASTRAL and MPEST, summaries of the results are presented for each of the five alignments. The three key relationships examined in the study, subfamily relationships, monophyly of Coregonus, and the sister lineage of Parahucho are presented along with support values if available. 


\begin{tabular}{|c|c|c|c|c|c|c|c|c|}
\hline \multirow{4}{*}{ StarBEAST2 Analyses } & Alignment & $\begin{array}{l}\text { Number of Independently } \\
\text { Modeled Loci }\end{array}$ & Sister Subfamilies & $\begin{array}{c}\text { Sister Subfamilies } \\
\text { Posterior Probability }\end{array}$ & $\begin{array}{l}\text { Monophyly of } \\
\text { Coregonus? }\end{array}$ & $\begin{array}{l}\text { Support for } \\
\text { Monophyly of } \\
\text { Coregonus }\end{array}$ & Sister of Parahucho & $\begin{array}{c}\text { Support for } \\
\text { Sister of } \\
\text { Parhucho }\end{array}$ \\
\hline & $100 \%$ Coverage & 6 & Coregoninae + Thymallinae & 0.98 & No & - & Salmo & 0.99 \\
\hline & $95 \%$ Coverage & 13 & Coregoninae + Thymallinae & 0.50 & Yes & 0.45 & Salmo & 0.98 \\
\hline & $93 \%$ Coverage & 28 & Coregoninae + Thymallinae & 0.34 & No & - & Salmo & 0.99 \\
\hline \multirow{5}{*}{ ASTRAL Analyses } & $75 \%$ Coverage & 105 & Coregoninae + Thymallinae & 0.52 & Yes & 0.43 & Salmo & 0.98 \\
\hline & Tetrasomic Loci & 33 & Coregoninae + Salmoninae & 0.99 & Yes & 1.00 & Oncorhynchus & 0.38 \\
\hline & & & & & & & & \\
\hline & $100 \%$ Coverage & 6 & Salmoninae + Thymallinae & 0.42 & Yes & 0.74 & $\begin{array}{c}(\text { Salmo }+(\text { Oncorhynchus }+ \\
\text { Salvelinus }))\end{array}$ & 0.69 \\
\hline & $95 \%$ Coverage & 13 & Salmoninae + Thymallinae & 0.38 & Yes & 0.68 & $\begin{array}{c}(\text { Salmo }+(\text { Oncorhynchus }+ \\
\text { Salvelinus }))\end{array}$ & 0.68 \\
\hline \multirow{9}{*}{ MP-EST Analyses } & $93 \%$ Coverage & 28 & Salmoninae + Thymallinae & 0.57 & No & - & Salmo & 0.46 \\
\hline & $75 \%$ Coverage & 105 & Salmoninae + Thymallinae & 0.50 & No & - & (Oncorhynchus + Salvelinus) & 0.54 \\
\hline & Tetrasomic Loci & 33 & Coregoninae + Salmoninae & 0.91 & Yes & 0.53 & (Oncorhynchus + Salvelinus) & 0.85 \\
\hline & & & & & & & & \\
\hline & $100 \%$ Coverage & 6 & Coregoninae + Salmoninae & & Yes & & Oncorhynchus & \\
\hline & $95 \%$ Coverage & 13 & Salmoninae + Thymallinae & & Yes & & (Salmo + Oncorhynchus $)$ & \\
\hline & 93\% Coverage & 28 & Salmoninae + Thymallinae & & No & & Salmo & \\
\hline & $75 \%$ Coverage & 105 & Coregoninae + Thymallinae & & No & & Oncorhynchus & \\
\hline & Tetrasomic Loci & 33 & Coregoninae + Salmoninae & & No & & (Oncorhynchus + Salvelinus $)$ & \\
\hline
\end{tabular}




\section{Table 3 (on next page)}

Hypothesis Testing with the Multispecies Coalescent.

The testing of subfamily relationships either through Bayes factor (StarBEAST2) or through the $t$ - statistic (MP-EST, described in text) are shown. Partitioning and modeling of each of the five alignments is summarized along with the number of loci analyzed. Significance values are presented along with the $t$ - statistic and are in bold face if significant. 


\begin{tabular}{|c|c|c|c|c|c|c|c|c|c|}
\hline \multirow[t]{2}{*}{$\mathbf{A}$} & StarBEAST2 Analyses & $\begin{array}{l}\text { Partitioning and Modeling } \\
\text { Scheme }\end{array}$ & $\begin{array}{c}\text { Number of Independently } \\
\text { Modeled Loci }\end{array}$ & Bayes Factor $C+T$ vs $C+S$ & Bayes Factor $\mathbf{C}+\mathbf{T}$ vs $\mathbf{S}+\mathbf{T}$ & Bayes Factor $\mathbf{C}+\mathbf{S}$ vs $\mathbf{S}+\mathbf{T}$ & & & \\
\hline & $100 \%$ Coverage Alignment & By UCE, ModelGenerator & 6 & 131.47 & 108.84 & 0.82 & & & \\
\hline \multirow{9}{*}{ B } & 95\% Coverage Alignment & By UCE, ModelGenerator & 13 & 1.82 & 2.21 & 1.21 & & & \\
\hline & 93\% Coverage Alignment & By UCE, HKY $+\Gamma$ & 28 & 1.00 & 1.01 & 1.00 & & & \\
\hline & $75 \%$ Coverage Alignment & By UCE, $\mathrm{HKY}+\Gamma$ & 105 & 1.23 & 9.33 & 7.57 & & & \\
\hline & Tetrasomic Loci Alignment & By UCE, HKY $+\Gamma$ & 33 & 0.00 & 0.81 & 248.20 & & & \\
\hline & MP-EST Analyses & $\begin{array}{l}\text { Partitioning and Modeling } \\
\text { Scheme }\end{array}$ & $\begin{array}{c}\text { Number of Independently } \\
\text { Modeled Loci }\end{array}$ & $t$ - statistic CT vs CS & $p$ - value & $t$ - statistic CT vs ST & $p$ - value & $t$ - statistic CS vs ST & $p$-value \\
\hline & $100 \%$ Coverage Alignment & By UCE & 6 & 14.80 & 0.27 & 11.69 & 0.37 & 3.10 & 0.28 \\
\hline & 95\% Coverage Alignment & By UCE & 13 & $5.60 \times 10-5$ & 0.49 & 0.61 & 0.47 & 0.61 & 0.41 \\
\hline & 93\% Coverage Alignment & By UCE & 28 & 4.74 & 0.39 & 2.26 & 0.27 & 7.01 & 0.27 \\
\hline & $75 \%$ Coverage Alignment & By UCE & 105 & 11.25 & 0.28 & 10.58 & 0.18 & 0.67 & 0.36 \\
\hline & Tetrasomic Loci Alignment & By UCE & 33 & 130.18 & 0.06 & $8.10 \times 10-4$ & 0.45 & 130.18 & 0.05 \\
\hline
\end{tabular}

